\title{
Randomness-Efficient Curve Samplers
}

\author{
Zeyu Guo* \\ Computer Science Department \\ California Institute of Technology \\ Pasadena, CA 91125
}

October 15, 2018

\begin{abstract}
Curve samplers are sampling algorithms that proceed by viewing the domain as a vector space over a finite field, and randomly picking a low-degree curve in it as the sample. Curve samplers exhibit a nice property besides the sampling property: the restriction of low-degree polynomials over the domain to the sampled curve is still low-degree. This property is often used in combination with the sampling property and has found many applications, including PCP constructions, local decoding of codes, and algebraic PRG constructions.

The randomness complexity of curve samplers is a crucial parameter for its applications. It is known that (non-explicit) curve samplers using $O(\log N+\log (1 / \delta))$ random bits exist, where $N$ is the domain size and $\delta$ is the confidence error. The question of explicitly constructing randomness-efficient curve samplers was first raised in [TSU06] where they obtained curve samplers with near-optimal randomness complexity.

We present an explicit construction of low-degree curve samplers with optimal randomness complexity (up to a constant factor), sampling curves of degree $\left(m \log _{q}(1 / \delta)\right)^{O(1)}$ in $\mathbb{F}_{q}^{m}$. Our construction is a delicate combination of several components, including extractor machinery, limited independence, iterated sampling, and list-recoverable codes.
\end{abstract}

\section{Introduction}

Randomness has numerous uses in computer science, and sampling is one of its most classical applications: Suppose we are interested in the size of a particular subset $A$ lying in a large domain $D$. Instead of counting the size of $A$ directly by enumeration, one can randomly draw a small sample from $D$ and calculate the density of $A$ in the sample. The approximated density is guaranteed to be close to the true density with probability $1-\delta$ where $\delta$ is very small, known as the confidence error. This sampling technique is extremely useful both in practice and in theory.

One class of sampling algorithms, known as curve samplers, proceed by viewing the domain as a vector space over a finite field, and picking a random low-degree curve in it. Curve samplers exhibit the following nice property besides the sampling property: the restriction of low-degree polynomials over the domain to the sampled curve is still low-degree. This special property, combined with the sampling property, turns out to be useful in many settings, e.g local decoding of Reed-Muller codes and hardness amplification [STV01], PCP constructions [AS98, ALM+98, MR08], algebraic

\footnotetext{
*zguo@caltech.edu. Supported by NSF CCF-1116111, NSF CCF-1038578 and BSF 2010120.
} 
constructions of pseudorandom-generators [SU05, Uma03], extractor constructions [SU05, TSU06], and some pure complexity results (e.g. [SU06]).

The problem of constructing explicit low-degree curve samplers was raised in [TSU06]. More specifically, we are looking for low-degree curve samplers with small sample complexity (polylogrithmic in the domain size) and confidence error (polynomially small in the domain size), and we focus on minimizing the randomness complexity. The simplest way is picking a completely random low-degree curve whose sampling properties are guaranteed by tail bounds for limited independence. The randomness complexity of this method, however, is far from being optimal. The probabilistic method guarantees the existence of (non-explicit) low-degree curve samplers using $O(\log N+\log (1 / \delta))$ random bits where $N$ is the domain size and $\delta$ is the confidence error. The real difficulty, however, is to find an explicit construction matching this bound.

\subsection{Previous work}

Randomness-efficient samplers (without the requirement that the sample points form a curve) are constructed in [CG89, Gil98, BR94, Zuc97]. In particular, [Zuc97] obtains explicit samplers with optimal randomness complexity (up to a $1+\gamma$ factor for arbitrary small $\gamma>0$ ) using the connection between samplers and extractors. See [Gol11] for a survey of samplers.

Degree-1 curve samplers are also called line samplers. Explicit randomness efficient line samplers are constructed in the PCP literature [BSSVW03, MR08], motivated by the goal of constructing almost linear sized PCPs. In [BSSVW03] line samplers are derandomized by picking a random point and a direction sampled from an $\epsilon$-biased set, instead of two random points. An alternative way is suggested in [MR08] where directions are picked from a subfield. It is not clear, however, how to apply these techniques to higher degree curves.

In [TSU06] it was shown how to explicitly construct derandomized curve samplers with nearoptimal parameters. Formally they obtained

- curve samplers picking curves of degree $(\log \log N+\log (1 / \delta))^{O(\log \log N)}$ using $O(\log N+$ $\log (1 / \delta) \log \log N)$ random bits, and

- curve samplers picking curves of degree $(\log (1 / \delta))^{O(1)}$ using $O\left(\log N+\log (1 / \delta)(\log \log N)^{1+\gamma}\right)$ random bits for any constant $\gamma>0$

for domain size $N$, field size $q \geq(\log N)^{\Theta(1)}$ and confidence error $\delta=N^{-\Theta(1)}$. Their work left the problem of explicitly constructing low-degree curve samplers (ideally picking curves of degree $\left.O\left(\log _{q}(1 / \delta)\right)\right)$ with essentially optimal $O(\log N+\log (1 / \delta))$ random bits as a prominent open problem.

\subsection{Main results}

We present an explicit construction of low-degree curve samplers with optimal randomness complexity (up to a constant factor). In particular, we show how to sample degree- $\left(m \log _{q}(1 / \delta)\right)^{O(1)}$ curves in $\mathbb{F}_{q}^{m}$ using $O(\log N+\log (1 / \delta))$ random bits for domain size $N=\left|\mathbb{F}_{q}^{m}\right|$ and confidence error $\delta=N^{-\Theta(1)}$. Before stating our main theorem, we first present the formal definition of samplers and curve samplers. 
Samplers. Given a finite set $\mathcal{M}$ as the domain, the density of a subset $A \subseteq \mathcal{M}$ is $\mu(A) \stackrel{\text { def }}{=} \frac{|A|}{|\mathcal{M}|}$. For a collection of elements $\mathcal{T}=\left\{t_{i}: i \in I\right\} \in \mathcal{M}^{I}$ indexed by set $I$, the density of $A$ in $\mathcal{T}$ is $\mu_{\mathcal{T}}(A) \stackrel{\text { def }}{=} \frac{|A \cap \mathcal{T}|}{|\mathcal{T}|}=\operatorname{Pr}_{i \in I}\left[t_{i} \in A\right]$

Definition 1.1 (sampler). A sampler is a function $S: \mathcal{N} \times \mathcal{D} \rightarrow \mathcal{M}$ where $|\mathcal{D}|$ is its sample complexity and $\mathcal{M}$ is its domain. We say $S$ samples $A \subseteq \mathcal{M}$ with accuracy error $\epsilon$ and confidence error $\delta$ if $\operatorname{Pr}_{x \in \mathcal{N}}\left[\left|\mu_{S(x)}(A)-\mu(A)\right|>\epsilon\right] \leq \delta$ where $S(x) \stackrel{\text { def }}{=}\{S(x, y): y \in \mathcal{D}\}$. We say $S$ is an $(\epsilon, \delta)$ sampler if it samples all subsets $A \subseteq \mathcal{M}$ with accuracy error $\epsilon$ and confidence error $\delta$. The randomness complexity of $S$ is $\log (|\mathcal{N}|)$.

Definition 1.2 (curve/line sampler). Let $\mathcal{M}=\mathbb{F}_{q}^{D}$ and $\mathcal{D}=\mathbb{F}_{q}$. The sampler $S: \mathcal{N} \times \mathcal{D} \rightarrow \mathcal{M}$ is a degree-t curve sampler if for all $x \in \mathcal{N}$, the function $S(x, \cdot): \mathcal{D} \rightarrow \mathcal{M}$ is a curve (see Definition 2.1) of degree at most $t$ over $\mathbb{F}_{q}$. When $t=1, S$ is also called a line sampler.

Theorem 1.1 (main). For any $\epsilon, \delta>0$, integer $m \geq 1$, and sufficiently large prime power $q \geq\left(\frac{m \log (1 / \delta)}{\epsilon}\right)^{\Theta(1)}$, there exists an explicit degree-t curve sampler for the domain $\mathbb{F}_{q}^{m}$ with $t=$ $\left(m \log _{q}(1 / \delta)\right)^{O(1)}$, accuracy error $\epsilon$, confidence error $\delta$, sample complexity $q$, and randomness complexity $O(m \log q+\log (1 / \delta))=O(\log N+\log (1 / \delta))$ where $N=q^{m}$ is the domain size. Moreover, the curve sampler itself has degree $\left(m \log _{q}(1 / \delta)\right)^{O(1)}$ as a polynomial map.

Theorem 1.1 has better degree bound and randomness complexity compared with the constructions in [TSU06]. We remark that the degree bound, being $\left(m \log _{q}(1 / \delta)\right)^{O(1)}$, is still sub-optimal compared with the lower bound $\log _{q}(1 / \delta)$ (see Appendix B for the proof of this lower bound). However in many cases it is satisfying to achieve such a degree bound.

As an example, consider the following setting of parameters: domain size $N=q^{m}$, field size $q=(\log N)^{\Theta(1)}$, confidence error $\delta=N^{-\Theta(1)}$, and accuracy error $\epsilon=(\log N)^{-\Theta(1)}$. Note that this is the typical setting in PCP and other literature [ALM $\left.{ }^{+} 98, \mathrm{AS} 98, \mathrm{STV} 01, \mathrm{SU} 05\right]$. In this setting, we have the following corollary in which the randomness complexity is logarithmic and the degree is polylogarithmic.

Corollary 1.1. Given domain size $N=\left|\mathbb{F}_{q}^{m}\right|$, accuracy error $\epsilon=(\log N)^{-\Theta(1)}$, confidence error $\delta=$ $N^{-\Theta(1)}$, and large enough field size $q=(\log N)^{\Theta(1)}$, there exists an explicit degree-t curve sampler for the domain $\mathbb{F}_{q}^{m}$ with accuracy error $\epsilon$, confidence error $\delta$, randomness complexity $O(\log N)$, sample complexity $q$, and $t \leq(\log N)^{c}$ for some constant $c>0$ independent of the field size $q$.

It remains an open problem to explicitly construct curve samplers that have optimal randomness complexity $O(\log N+\log (1 / \delta)$ ) (up to a constant factor), and sample curves with optimal degree bound $O\left(\log _{q}(1 / \delta)\right)$. It is also an interesting problem to achieve the optimal randomness complexity up to a $1+\gamma$ factor for any constant $\gamma>0$ (rather than just an $O(1)$ factor), as achieved by [Zuc97] for general samplers. The standard techniques as in [Zuc97] are not directly applicable as they increase the dimension of samples and only yield $O(1)$-dimensional manifold samplers.

\subsection{Techniques}

Extractor machinery. It was shown in [Zuc97] that samplers are equivalent to extractors, objects that convert weakly random distributions into almost uniform distributions. Therefore the 
techniques of constructing extractors are extremely useful in constructing curve samplers. Our construction employs the technique of block source extraction [NZ96, Zuc97, SZ99]. In addition, we also use the techniques appeared in [GUV09], especially their constructions of condensers.

Limited independence. It is well known that points on a random degree- $(t-1)$ curve are $t$-wise independent. So we may simply pick a random curve and use tail inequalities to bound the confidence error. However, the sample complexity is too high, and hence we need to use the technique of iterated sampling to reduce the number of sample points.

Iterated sampling. Iterated sampling is a useful technique for constructing explicit randomnessefficient samplers [BR94, TSU06]. The idea is first picking a large sample from the domain and then draw a sub-sample from the previous sample. The drawback of iterated sampling, however, is that it invests randomness twice while the confidence error does not shrink correspondingly. To remedy this problem, we add another ingredient into our construction, namely the technique of error reduction.

Error reduction via list-recoverable codes. We will use explicit list-recoverable codes [GI01], a strengthening of list-decodable codes. More specifically, we will employ the list-recoverability from (folded) Reed-Solomon codes [GR08, GUV09]. List-recoverable codes provide a way of obtaining samplers with very small confidence error from those with mildly small confidence error. We refer to this transformation as error reduction, which plays a key role in our construction.

\subsection{Sketch of the construction}

Our curve sampler is the composition of two samplers which we call the outer sampler and the inner sampler respectively. The outer sampler picks manifolds (see Definition 2.1) of dimension $O(\log m$ ) from the domain $\mathcal{M}=\mathbb{F}_{q}^{m}$. The outer sampler has near-optimal randomness complexity but the sample complexity is large. To fix this problem, we employ the idea of iterated sampling. Namely we regard the manifold picked by the outer sampler as the new domain $\mathcal{M}^{\prime}$, and then construct an inner sampler picking a curve from $\mathcal{M}^{\prime}$ with small sample complexity.

The outer sampler is obtained by constructing an extractor and then using the extractor-sampler connection [Zuc97]. We follow the approach in [NZ96, Zuc97, SZ99]: Given an arbitrary random source with enough min-entropy, we will first use a block source converter to convert it into a block source, and then feed it to a block source extractor. In addition, we need to construct these components carefully so as to maintain the low-degree-ness. The way we construct the block source converter is different from those in [NZ96, Zuc97, SZ99] (as they are not in the form of low-degree polynomial maps), and is based on the Reed-Solomon condenser proposed in [GUV09]: To obtain one block, we simply feed the random source and a fresh new seed into the condenser, and let the output be the block. We show that this indeed gives a block source.

The inner sampler is constructed using techniques of iterated sampling and error reduction. We start with the basic curve samplers picking totally random curves, and then apply the error reduction as well as iterated sampling techniques repeatedly to obtain the desired inner sampler. Either of the two operations improves one parameter while worsening some other one: Iterated sampling reduces sample complexity but increases the randomness complexity, whereas error reduction reduces the confidence error but increases the sample complexity. Our construction applies the 
two techniques alternately such that (1) we keep the invariant that the confidence error is always exponentially small in the randomness complexity, and (2) the sample complexity is finally brought down to $q$.

Outline. The next section contains relevant definitions and some basic facts. Section 3 gives the construction of the outer sampler using block source extraction. Section 4 introduces the techniques of error reduction and iterated sampling, and then uses them to construct the inner sampler. These components are finally put together in Section 5 and yield the curve sampler construction.

\section{Preliminaries}

We denote the set of numbers $\{1,2, \ldots, n\}$ by $[n]$. Given a prime power $q$, write $\mathbb{F}_{q}$ for the finite field of size $q$. Write $U_{n, q}$ for the uniform distribution over $\mathbb{F}_{q}^{n}$. Logarithms are taken with base 2 unless the base is explicitly specified.

Random variables and distributions are represented by upper-case letters whereas their specific values are represented by lower-case letters. Write $x \leftarrow X$ if $x$ is sampled according to distribution $X$. The support of a distribution $X$ over set $S$ is $\operatorname{supp}(X) \stackrel{\text { def }}{=}\{x \in S: \operatorname{Pr}[X=x]>0\}$. The statistical distance between distributions $X, Y$ over set $S$ is defined as $\Delta(X, Y)=\max _{T \subseteq S} \mid \operatorname{Pr}[X \in$ $T]-\operatorname{Pr}[Y \in T] \mid$. We say $X$ is $\epsilon$-close to $Y$ if $\Delta(X, Y) \leq \epsilon$.

For an event $A$, let $\mathbf{I}[A]$ be the indicator variable that evaluates to 1 if $A$ occurs and 0 otherwise. For a random variable $X$ and an event $A$ that occurs with nonzero probability, define the conditional distribution $\left.X\right|_{A}$ by $\operatorname{Pr}\left[\left.X\right|_{A}=x\right]=\frac{\operatorname{Pr}[(X=x) \wedge A]}{\operatorname{Pr}[A]}$.

Manifolds and curves. Let $f: \mathbb{F}_{q}^{d} \rightarrow \mathbb{F}_{q}^{D}$ be a polynomial map. We may view $f$ as $D$ individual polynomials $f_{i}: \mathbb{F}_{q}^{d} \rightarrow \mathbb{F}_{q}$ describing its operation on each output coordinate, i.e., $f(x)=\left(f_{1}(x), \ldots, f_{D}(x)\right)$ for all $x \in \mathbb{F}_{q}^{d}$. Such maps are called curves or manifolds, depending on the dimension $d$.

Definition 2.1 (manifold). A manifold in $\mathbb{F}_{q}^{D}$ is a polynomial map $M: \mathbb{F}_{q}^{d} \rightarrow \mathbb{F}_{q}^{D}$ where $M_{1}, \ldots, M_{D}$ are $d$-variate polynomials over $\mathbb{F}_{q}$. We call $d$ the dimension of $M$. An 1-dimensional manifold is also called a curve. A curve of degree 1 is also called a line. The degree of $M$ is $\operatorname{deg}(M) \stackrel{\text { def }}{=}$ $\left.\max \left\{\operatorname{deg}\left(M_{1}\right), \ldots, \operatorname{deg}\left(M_{D}\right)\right)\right\}$.

We need the following lemma, generalizing the one in [TSU06]. Its proof is deferred to the appendix.

Lemma 2.1. A manifold $f:\left(\mathbb{F}_{q}\right)^{n} \rightarrow\left(\mathbb{F}_{q^{D}}\right)^{m}$ of degree $t$, when viewed as a manifold $f:\left(\mathbb{F}_{q}^{D}\right)^{n} \rightarrow$ $\left(\mathbb{F}_{q}^{D}\right)^{m}$, also has degree at most $t$.

Basic line/curve samplers The simplest line (resp. curve) samplers are those picking completely random lines (resp. curves), as defined below. We call them basic line (resp. curve) samplers.

Definition 2.2 (basic line sampler). For $m \geq 1$ and prime power $q$, let Line L $_{m, q}: \mathbb{F}_{q}^{2 m} \times \mathbb{F}_{q} \rightarrow \mathbb{F}_{q}^{m}$ be the line sampler that picks a completely random line in $\mathbb{F}_{q}^{m}$. Formally,

$$
\text { Line }_{m, q}((a, b), y) \stackrel{\text { def }}{=}\left(a_{1} y+b_{1}, \ldots, a_{m} y+b_{m}\right)
$$


for $a=\left(a_{1}, \ldots, a_{m}\right), b=\left(b_{1}, \ldots, b_{m}\right) \in \mathbb{F}_{q}^{m}$ and $y \in \mathbb{F}_{q}$.

Definition 2.3 (basic curve sampler). For $m \geq 1, t \geq 4$ and prime power $q$, let Curve lut, $_{\text {a }}$ : $\mathbb{F}_{q}^{t m} \times \mathbb{F}_{q} \rightarrow \mathbb{F}_{q}^{m}$ be the curve sampler that picks a completely random curve of degree $t-1$ in $\mathbb{F}_{q}^{m}$. Formally,

$$
\text { Curve }_{m, t, q}\left(\left(c_{0}, \ldots, c_{t-1}\right), y\right) \stackrel{\text { def }}{=}\left(\sum_{i=0}^{t-1} c_{i, 1} y^{i}, \ldots, \sum_{i=0}^{t-1} c_{i, m} y^{i}\right)
$$

for each $c_{0}=\left(c_{0,1}, \ldots, c_{0, m}\right), \ldots, c_{t-1}=\left(c_{t-1,1}, \ldots, c_{t-1, m}\right) \in \mathbb{F}_{q}^{m}$ and $y \in \mathbb{F}_{q}$.

Remark 1. Note that Line $_{m, q}$ has degree 2 and Curve $_{m, t, q}$ has degree $t$ as polynomial maps.

Tail inequalities for limited independent random variables imply the following lemmas stating that basic line/curve samplers are indeed good samplers.

Lemma 2.2. For $\epsilon>0, m \geq 1$ and prime power $q$, Line L $_{, q}$ is an $\left(\epsilon, \frac{1}{\epsilon^{2} q}\right)$ line sampler.

Lemma 2.3. For $\epsilon>0, m \geq 1, t \geq 4$ and sufficiently large prime power $q=(t / \epsilon)^{O(1)}$, Curve $_{m, t, q}$ is an $\left(\epsilon, q^{-t / 4}\right)$ sampler.

See the appendix for the proofs of Lemma 2.2 and Lemma 2.3.

Extractors and condensers. A (seeded) extractor is an object that takes an imperfect random variable called the (weakly) random source, invests a small amount of randomness called the seed, and produces an output whose distribution is very close to the uniform distribution.

Definition 2.4 ( $q$-ary min-entropy). We say $X$ has $q$-ary min-entropy $k$ if for any $x \in S$, it holds that $\operatorname{Pr}[X=x] \leq q^{-k}$ (or equivalently, $X$ has min-entropy $k \log q$ ).

Definition 2.5 (condenser/extractor). Given a function $f: \mathbb{F}_{q}^{n} \times \mathbb{F}_{q}^{d} \rightarrow \mathbb{F}_{q}^{m}$, we say $f$ is an $k_{1} \rightarrow_{\epsilon, q} k_{2}$ condenser if for every distribution $X$ with $q$-ary min-entropy $k_{1}, f\left(X, U_{d, q}\right)$ is $\epsilon$-close to a distribution with $q$-ary min-entropy $k_{2}$. We say $f$ is a $(k, \epsilon, q)$ extractor if it is a $k \rightarrow_{\epsilon, q} m$ condenser.

Remark 2. We are interested in extractors and samplers that are polynomial maps. For such an object $f$, we denote by $\operatorname{deg}(f)$ its degree as a polynomial map.

The following connection between extractors and samplers was observed in [Zuc97].

Theorem 2.1 ([Zuc97], restated). Given a map $f: \mathbb{F}_{q}^{n} \times \mathbb{F}_{q}^{d} \rightarrow \mathbb{F}_{q}^{m}$, we have the following:

1. If $f$ is a $(k, \epsilon, q)$ extractor, then it is also an $(\epsilon, \delta)$ sampler where $\delta=2 q^{k-n}$.

2. If $f$ is an $(\epsilon / 2, \delta)$ sampler where $\delta=\epsilon q^{k-n}$, then it is also a $(k, \epsilon, q)$ extractor.

See the appendix for the proof. 


\section{Outer sampler}

In this section we construct a sampler whose randomness complexity is optimal up to a constant factor. We refer to it as the "outer sampler".

We need the machinery of block source extraction.

Definition 3.1 (block source [CG88]). A random source $X=\left(X_{1}, \ldots, X_{s}\right)$ over $\mathbb{F}_{q}^{n_{1}} \times \cdots \times \mathbb{F}_{q}^{n_{s}}$ is a $\left(k_{1}, \ldots, k_{s}\right) q$-ary block source if for any $i \in[s]$ and $\left(x_{1}, \ldots, x_{i-1}\right) \in \operatorname{supp}\left(X_{1}, \ldots, X_{i-1}\right)$, the conditional distribution $\left.X_{i}\right|_{X_{1}=x_{1}, \ldots, X_{i-1}=x_{i-1}}$ has $q$-ary min-entropy $k_{i}$. Each $X_{i}$ is called a block.

Definition 3.2 (block source extractor). A function $E:\left(\mathbb{F}_{q}^{n_{1}} \times \cdots \times \mathbb{F}_{q}^{n_{s}}\right) \times \mathbb{F}_{q}^{d} \rightarrow \mathbb{F}_{q}^{m}$ is called a $\left(\left(k_{1}, \ldots, k_{s}\right), \epsilon, q\right)$ block source extractor if for any $\left(k_{1}, \ldots, k_{s}\right) q$-ary block source $\left(X_{1}, \ldots, X_{s}\right)$ over $\mathbb{F}_{q}^{n_{1}} \times \cdots \times \mathbb{F}_{q}^{n_{s}}$, the distribution $E\left(\left(X_{1}, \ldots, X_{s}\right), U_{d, q}\right)$ is $\epsilon$-close to $U_{m, q}$.

One nice property of block sources is that their special structure allows us to compose several extractors and get a block source extractor with only a small amount of randomness invested.

Definition 3.3 (block source extraction via composition). Let $s \geq 1$ be an integer and $E_{i}$ : $\mathbb{F}_{q}^{n_{i}} \times \mathbb{F}_{q}^{d_{i}} \rightarrow \mathbb{F}_{q}^{m_{i}}$ be a map for each $i \in[s]$. Suppose that $m_{i} \geq d_{i-1}$ for all $i \in[s]$, where we set $d_{0}=0$. Define $E=\operatorname{BlkExt}\left(E_{1}, \ldots, E_{s}\right)$ as follows:

$$
\begin{aligned}
E:\left(\mathbb{F}_{q}^{n_{1}} \times \cdots \times \mathbb{F}_{q}^{n_{s}}\right) \times \mathbb{F}_{q}^{d_{s}} & \rightarrow\left(\mathbb{F}_{q}^{m_{1}-d_{0}} \times \cdots \times \mathbb{F}_{q}^{m_{s}-d_{s-1}}\right) \\
\left(\left(x_{1}, \ldots, x_{s}\right), y_{s}\right) & \mapsto\left(z_{1}, \ldots, z_{s}\right)
\end{aligned}
$$

where for $i=s, \ldots, 1$, we iteratively define $\left(y_{i-1}, z_{i}\right)$ to be a partition of $E_{i}\left(x_{i}, y_{i}\right)$ into the prefix $y_{i-1} \in \mathbb{F}_{q}^{d_{i-1}}$ and the suffix $z_{i} \in \mathbb{F}_{q}^{m_{i}-d_{i-1}}$.

See Figure 1 for an illustration of the above definition.

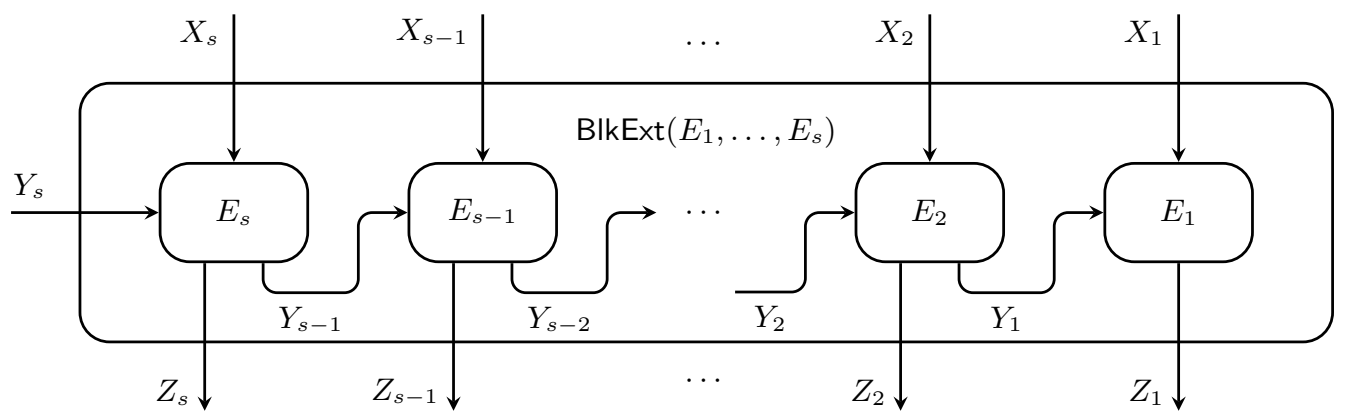

Figure 1: The composed block source extractor $\operatorname{BlkExt}\left(E_{1}, \ldots, E_{s}\right)$.

Lemma 3.1. Let $s \geq 1$ be an integer and for each $i \in[s]$, let $E_{i}: \mathbb{F}_{q}^{n_{i}} \times \mathbb{F}_{q}^{d_{i}} \rightarrow \mathbb{F}_{q}^{m_{i}}$ be $a\left(k_{i}, \epsilon_{i}, q\right)$ extractor of degree $t_{i} \geq 1$. Then $\operatorname{BlkExt}\left(E_{1}, \ldots, E_{s}\right)$ is a $\left(\left(k_{1}, \ldots, k_{s}\right), \epsilon, q\right)$ block source extractor of degree $t$ where $\epsilon=\sum_{i=1}^{s} \epsilon_{i}$ and $t=\prod_{i=1}^{s} t_{i}$.

The proof is deferred to the appendix. 


\subsection{Block source conversion}

Definition 3.4 (block source converter [NZ96]). A function $C: \mathbb{F}_{q}^{n} \times \mathbb{F}_{q}^{d} \rightarrow \mathbb{F}_{q}^{m_{1}} \times \cdots \times \mathbb{F}_{q}^{m_{s}}$ is a $\left(k,\left(k_{1}, \ldots, k_{s}\right), \epsilon, q\right)$ block source converter if for any random source $X$ over $\mathbb{F}_{q}^{n}$ with $q$-ary min-entropy $k$, the output $C\left(X, U_{d, q}\right)$ is $\epsilon$-close to a $\left(k_{1}, \ldots, k_{s}\right) q$-ary block source.

It was shown in [NZ96] that one can obtain a block by choosing a pseudorandom subset of bits of the random source. Yet the analysis is pretty delicate and cumbersome. Furthermore the resulting extractor does not have a nice algebraic structure. We observe that the following condenser from Reed-Solomon codes in [GUV09] can be used to obtain blocks and is a low-degree manifold.

Definition 3.5 (condenser from Reed-Solomon codes [GUV09]). Let $\zeta \in \mathbb{F}_{q}$ be a generator of the multiplicative group $\mathbb{F}_{q}^{\times}$. Define $\operatorname{RSCon}_{n, m, q}: \mathbb{F}_{q}^{n} \times \mathbb{F}_{q} \rightarrow \mathbb{F}_{q}^{m}$ for $n, m \geq 1$ and prime power $q$ :

$$
\operatorname{RSCon}_{n, m, q}(x, y)=\left(y, f_{x}(y), f_{x}(\zeta y), \ldots, f_{x}\left(\zeta^{m-2} y\right)\right)
$$

where $f_{x}(Y)=\sum_{i=0}^{n-1} x_{i} Y^{i}$ for $x=\left(x_{0}, x_{1}, \ldots, x_{n-1}\right) \in \mathbb{F}_{q}^{n}$.

Theorem 3.1 ([GUV09]). RSCon ${ }_{n, m, q}$ is a $m \rightarrow_{\epsilon, q} 0.99 m$ condenser for large enough $q \geq(n / \epsilon)^{O(1)}$.

Remark 3. The condenser $\operatorname{RSCon}_{n, m, q}(x, y)$ is a degree- $n$ manifold, as each monomial in any of its coordinate is of the form $y$ or $x_{i}\left(\zeta^{j} y\right)^{i}$ for some $0 \leq i \leq n-1$.

We apply the above condenser to the source with independent seeds to obtain a block source.

Definition 3.6 (block source converter via condensing). For integers $n, m_{1}, \ldots, m_{s} \geq 1$ and prime power $q$, define the function BlkCnvt ${\text { B },\left(m_{1}, \ldots, m_{s}\right), q}: \mathbb{F}_{q}^{n} \times \mathbb{F}_{q}^{s} \rightarrow \mathbb{F}_{q}^{m_{1}+\cdots+m_{s}}$ by

$$
\mathrm{BlkCnvt}_{n,\left(m_{1}, \ldots, m_{s}\right), q}(x, y)=\left(\operatorname{RSCon}_{n, m_{1}, q}\left(x, y_{1}\right), \ldots, \operatorname{RSCon}_{n, m_{s}, q}\left(x, y_{s}\right)\right)
$$

for $x \in \mathbb{F}_{q}^{n}$ and $y=\left(y_{1}, \ldots, y_{s}\right) \in \mathbb{F}_{q}^{s}$.

The function BlkCnvt ${ }_{n,\left(m_{1}, \ldots, m_{s}\right), q}$ is indeed a block source converter. The intuition is that conditioning on the values of the previous blocks, the random source $X$ still has enough minentropy, and hence we may apply the condenser to get the next block. Formally, we have the following statement whose proof is deferred to the appendix.

Theorem 3.2. For $\epsilon>0$, integers $s, n, m_{1}, \ldots, m_{s} \geq 1$ and sufficiently large prime power $q=$ $(n / \epsilon)^{O(1)}$, the function BlkCnvt $_{n,\left(m_{1}, \ldots, m_{s}\right), q}$ is a $\left(k,\left(k_{1}, \ldots, k_{s}\right), 3 s \epsilon, q\right)$ block source converter of degree $n$ where $k=\sum_{i=1}^{s} m_{i}+\log _{q}(1 / \epsilon)$ and each $k_{i}=0.99 m_{i}$.

\subsection{Construction of the outer sampler}

By Lemma 2.2 and Theorem 2.1, the basic line samplers are also extractors.

Lemma 3.2. For $\epsilon>0, m \geq 1$ and prime power $q$, Line $m, q$ is $a(k, \epsilon, q)$ extractor of degree 2 where $k=2 m-1+3 \log _{q}(1 / \epsilon)$.

We employ Lemma 3.1 and compose the basic line samplers to get a block source extractor. It is then applied to a block source obtained from the block source converter. 
Definition 3.7 (Outer Sampler). For $\delta>0, m=2^{s}$ and prime power $q$, let $n=4 m+\left\lceil\log _{q}(2 / \delta)\right\rceil$, $d=s+1$, and $d_{i}=2^{s-i}$ for $i \in[s]$. For $i \in[s]$, view Line $_{2, q^{d_{i}}}: \mathbb{F}_{q^{d_{i}}}^{4} \times \mathbb{F}_{q^{d_{i}}} \rightarrow \mathbb{F}_{q^{d_{i}}}^{2}$ as a manifold over $\mathbb{F}_{q}$ : Line $_{2, q_{i} d_{i}}: \mathbb{F}_{q}^{4 d_{i}} \times \mathbb{F}_{q}^{d_{i}} \rightarrow \mathbb{F}_{q}^{2 d_{i}}$. Composing these line samplers Line ${ }_{2, q_{i}}$ for $i \in[s]$ gives the function BlkExt $\left(\right.$ Line $_{2, q^{d_{1}}}, \ldots$, Line $\left._{2, q^{d_{s}}}\right): \mathbb{F}_{q}^{4 d_{1}+\cdots+4 d_{s} \times \mathbb{F}_{q} \rightarrow \mathbb{F}_{q}^{m} \text {. Finally, define OuterSamp } m, \delta, q}$ : $\mathbb{F}_{q}^{n} \times \mathbb{F}_{q}^{d} \rightarrow \mathbb{F}_{q}^{m}$

OuterSamp $_{m, \delta, q}\left(x,\left(y, y^{\prime}\right)\right) \stackrel{\text { def }}{=} \operatorname{BlkExt}\left(\operatorname{Line}_{2, q^{d_{1}}}, \ldots, \operatorname{Line}_{2, q^{d_{s}}}\right)\left(\operatorname{BlkCnvt}_{n,\left(4 d_{1}, \ldots, 4 d_{s}\right), q}(x, y), y^{\prime}\right)$

for $x \in \mathbb{F}_{q}^{n}, y \in \mathbb{F}_{q}^{s}$ and $y^{\prime} \in \mathbb{F}_{q}$.

See Figure 2 for an illustration of the above construction.

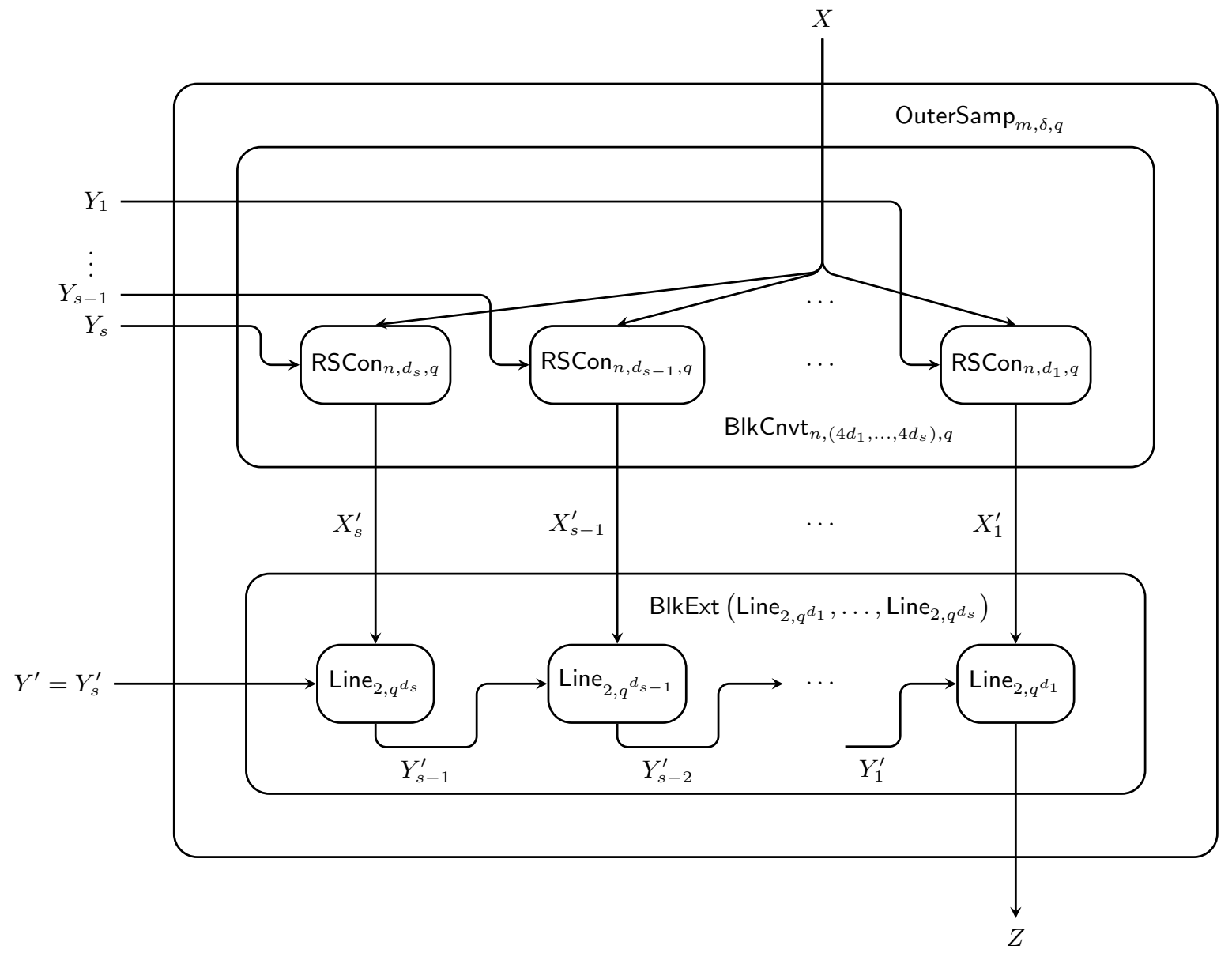

Figure 2: The outer sampler OuterSamp $m, \delta, q$.

Theorem 3.3. For any $\epsilon, \delta>0$, integer $m \geq 1$, and sufficiently large prime power $q \geq(n / \epsilon)^{O(1)}$, OuterSamp $_{m, \delta, q}$ is an $(\epsilon, \delta)$ sampler of degree $t$ where $d=O(\log m), n=O\left(m+\log _{q}(1 / \delta)\right)$ and $t=O\left(m^{2}+m \log _{q}(1 / \delta)\right)$.

Theorem 3.3 is proven by combining Lemma 2.1, Theorem 2.1, Lemma 3.1, Theorem 3.2, and Lemma 3.2. We defer the proof to the appendix. 
Remark 4. We assume $m$ is a power of 2 above. For general $m$, simply pick $m^{\prime}=2^{\lceil\log m\rceil}$ and let OuterSamp $_{m, \delta, q}$ be the composition of OuterSamp $m^{\prime}, \delta, q$ with the projection $\pi: \mathbb{F}_{q}^{m^{\prime}} \rightarrow \mathbb{F}_{q}^{m}$ onto the first $m$ coordinates. It yields an $(\epsilon, \delta)$ sampler of degree $t$ for $\mathbb{F}_{q}^{m}$ since $\pi$ is linear, and approximating the density of a subset $A$ in $\mathbb{F}_{q}^{m}$ is equivalent to approximating the density of $\pi^{-1}(A)$ in $\mathbb{F}_{q}^{m^{\prime}}$.

\section{Inner sampler}

The sampler OuterSamp $m, \delta, q$ has randomness complexity $O(m \log q+\log (1 / \delta))$ which is optimal up to a constant factor. Yet the sample complexity is large, being $q^{O(\log m)}$. We remedy this problem by composing it with an "inner sampler" with small sample complexity. Its construction is based on two techniques called error reduction and iterated sampling.

\subsection{Error reduction}

Condensers are at the core of many extractor constructions [RSW06, TSUZ07, GUV09, TSU12]. In the language of samplers, the use of condensers can be regarded as an error reduction technique, as we shall see below.

Given a function $f: \mathbb{F}_{q}^{n} \times \mathbb{F}_{q}^{d} \rightarrow \mathbb{F}_{q}^{m}$, define $\operatorname{LIST}_{f}(T, \epsilon) \stackrel{\text { def }}{=}\left\{x \in \mathbb{F}_{q}^{n}: \operatorname{Pr}_{y}[f(x, y) \in T]>\epsilon\right\}$ for any $T \subseteq \mathbb{F}_{q}^{m}$ and $\epsilon>0$. We are interesting in functions $f$ exhibiting a "list-recoverability" property that the size of $\operatorname{LIST}_{f}(T, \epsilon)$ is kept small when $T$ is not too large.

Definition 4.1. A function $f: \mathbb{F}_{q}^{n} \times \mathbb{F}_{q} \rightarrow \mathbb{F}_{q}^{m}$ is $(\epsilon, L, H)$ list-recoverable if $\left|\operatorname{LIST}_{f}(T, \epsilon)\right| \leq H$ for all $T \subseteq \mathbb{F}_{q}^{m}$ of size at most $L$.

We then define an operation $\star$ as follows.

Definition 4.2. For functions $f: \mathbb{F}_{q}^{n} \times \mathbb{F}_{q}^{d} \rightarrow \mathbb{F}_{q}^{m}$ and $S: \mathbb{F}_{q}^{m} \times \mathbb{F}_{q}^{d^{\prime}} \rightarrow \mathbb{F}_{q}^{m^{\prime}}$, define $S \star f:$ $\mathbb{F}_{q}^{n} \times\left(\mathbb{F}_{q}^{d} \times \mathbb{F}_{q}^{d^{\prime}}\right) \rightarrow \mathbb{F}_{q}^{m^{\prime}}$ such that $(S \star f)\left(x,\left(y, y^{\prime}\right)\right) \stackrel{\text { def }}{=} S\left(f(x, y), y^{\prime}\right)$.

See Figure 3 for an illustration. The following lemma states that a sampler with mildly small confidence error, when composed with a list-recoverable function via the $\star$ operation, gives a sampler with very small confidence error.

Lemma 4.1. Suppose $f: \mathbb{F}_{q}^{n} \times \mathbb{F}_{q}^{d} \rightarrow \mathbb{F}_{q}^{m}$ is $\left(\epsilon_{1}, L, H\right)$ list-recoverable, and $S: \mathbb{F}_{q}^{m} \times \mathbb{F}_{q}^{d^{\prime}} \rightarrow \mathbb{F}_{q}^{m^{\prime}}$ is an $\left(\epsilon_{2}, L / q^{m}\right)$ sampler. Then $S \star f$ is an $\left(\epsilon_{1}+\epsilon_{2}, H / q^{n}\right)$ sampler.

Proof. Let $A$ be an arbitrary subset of $\mathbb{F}_{q}^{m^{\prime}}$. Let $B=\left\{y \in \mathbb{F}_{q}^{m}:\left|\mu_{S(y)}-\mu(A)\right|>\epsilon_{2}\right\}$. By the sampling property of $S$, we have $|B| \leq\left(L / q^{m}\right) \cdot q^{m}=L$ and hence $\left|\operatorname{LIST}_{f}\left(B, \epsilon_{1}\right)\right| \leq H$. Therefore it suffices to show that for any $x \in \mathbb{F}_{q}^{n} \backslash \operatorname{LIST}_{f}\left(B, \epsilon_{1}\right)$, it holds that $\left|\mu_{(S \star f)(x)}(A)-\mu(A)\right| \leq \epsilon_{1}+\epsilon_{2}$.

Fix $x \in \mathbb{F}_{q}^{n} \backslash \operatorname{LIST}_{f}\left(B, \epsilon_{1}\right)$. We have

$$
\mu_{(S \star f)(x)}(A)=\operatorname{Pr}_{y, y^{\prime}}\left[(S \star f)\left(x,\left(y, y^{\prime}\right)\right) \in A\right]=\operatorname{Pr}_{y, y^{\prime}}\left[S\left(f(x, y), y^{\prime}\right) \in A\right]=\mathbb{E}_{y}\left[\mu_{S(f(x, y))}(A)\right] .
$$

Therefore

$$
\begin{aligned}
\left|\mu_{(S \star f)(x)}(A)-\mu(A)\right|=\mid \mathbb{E}_{y}[ & \left.\mu_{S(f(x, y))}(A)\right]-\mu(A)\left|\leq \mathbb{E}_{y}\right| \mu_{S(f(x, y))}(A)-\mu(A) \mid \\
& \leq \operatorname{Pr}_{y}[f(x, y) \in B]+\epsilon_{2} \operatorname{Pr}_{y}[f(x, y) \notin B] \leq \epsilon_{1}+\epsilon_{2} .
\end{aligned}
$$

To see the last two steps, note that $\left|\mu_{S(y)}(A)-\mu(A)\right| \leq \epsilon_{2}$ for $y \notin B$ by definition, and $\operatorname{Pr}_{y}[f(x, y) \in$ $B] \leq \epsilon_{1}$ since $x \notin \operatorname{LIST}_{f}\left(B, \epsilon_{1}\right)$. 
The condenser $\mathrm{RSCon}_{n, m, q}$ (see Definition 3.5) enjoys the following list-recoverability property:

Theorem 4.1 ([GUV09]). RSCon ${ }_{n, m, q}$ is $\left(\epsilon, q^{0.99 m}, q^{m}\right)$ list-recoverable for sufficiently large $q \geq$ $(n / \epsilon)^{O(1)}$.

Corollary 4.1. For any $n \geq m \geq 1, \epsilon, \epsilon^{\prime}>0$ and sufficiently large prime power $q=(n / \epsilon)^{O(1)}$, suppose $S: \mathbb{F}_{q}^{m} \times \mathbb{F}_{q}^{d} \rightarrow \mathbb{F}_{q}^{m^{\prime}}$ is an $\left(\epsilon^{\prime}, q^{-0.01 m}\right)$ sampler of degree $t$, then $S \star \mathrm{RSCon}_{n, m, q}$ is an $\left(\epsilon+\epsilon^{\prime}, q^{m-n}\right)$ sampler of degree $n t$.

Proof. Apply Lemma 4.1 and Theorem 4.1. Note that RSCon $_{n, m, q}$ has degree $n$. Therefore $\left(S \star \operatorname{RSCon}_{n, m, q}\right)\left(X,\left(Y, Y^{\prime}\right)\right)=S\left(\operatorname{RSCon}_{n, m, q}(X, Y), Y^{\prime}\right)$ has degree $n t$ in its variables $X, Y, Y^{\prime}$.

\subsection{Iterated sampling}

We introduce the operation o denoting the composition of two samplers.

Definition 4.3. (composed sampler). Given functions $S_{1}: \mathbb{F}_{q}^{n_{1}} \times \mathbb{F}_{q}^{d_{1}} \rightarrow \mathbb{F}_{q}^{d_{0}}$ and $S_{2}: \mathbb{F}_{q}^{n_{2}} \times \mathbb{F}_{q}^{d_{2}} \rightarrow$ $\mathbb{F}_{q}^{d_{1}}$, define $S_{1} \circ S_{2}:\left(\mathbb{F}_{q}^{n_{1}} \times \mathbb{F}_{q}^{n_{2}}\right) \times \mathbb{F}_{q}^{d_{2}} \rightarrow \mathbb{F}_{q}^{d_{0}}$ such that $\left(S_{1} \circ S_{2}\right)\left(\left(x_{1}, x_{2}\right), y\right) \stackrel{\text { def }}{=} S_{1}\left(x_{1}, S_{2}\left(x_{2}, y\right)\right)$.

See Figure 4 for an illustration. The composed sampler $S_{1} \circ S_{2}$ first uses its randomness $x_{1}$ to get the sample $S_{1}\left(x_{1}\right)=\left\{S_{1}\left(x_{1}, y\right): y \in \mathbb{F}_{q}\right\}$, and then uses its randomness $x_{2}$ to get the subsample $\left\{S_{1}\left(x_{1}, S_{2}\left(x_{2}, y\right)\right): y \in \mathbb{F}_{q}\right\} \subseteq S_{1}\left(x_{1}\right)$. Intuitively, if $S_{1}$ and $S_{2}$ are good samplers then so is $S_{1} \circ S_{2}$. This is indeed shown by [BR94, TSU06] and we formalize it as follows:

Lemma 4.2 ([BR94, TSU06]). Let $S_{1}: \mathbb{F}_{q}^{n_{1}} \times \mathbb{F}_{q}^{d_{1}} \rightarrow \mathbb{F}_{q}^{d_{0}}$ be an $\left(\epsilon_{1}, \delta_{1}\right)$ sampler of degree $t_{1}$. And let $S_{2}: \mathbb{F}_{q}^{n_{2}} \times \mathbb{F}_{q}^{d_{2}} \rightarrow \mathbb{F}_{q}^{d_{1}}$ be an $\left(\epsilon_{2}, \delta_{2}\right)$ sampler of degree $t_{2}$. Then $S_{1} \circ S_{2}:\left(\mathbb{F}_{q}^{n_{1}} \times \mathbb{F}_{q}^{n_{2}}\right) \times \mathbb{F}_{q}^{d_{2}} \rightarrow \mathbb{F}_{q}^{d_{0}}$ is an $\left(\epsilon_{1}+\epsilon_{2}, \delta_{1}+\delta_{2}\right)$ sampler of degree $t_{1} t_{2}$.

See the appendix for its proof.

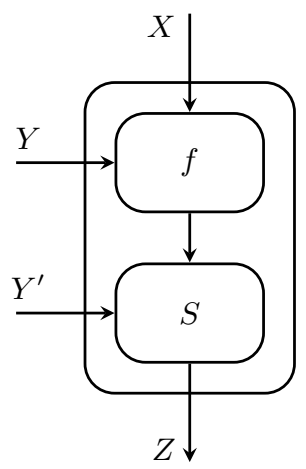

Figure 3: The operation $S \star f$.

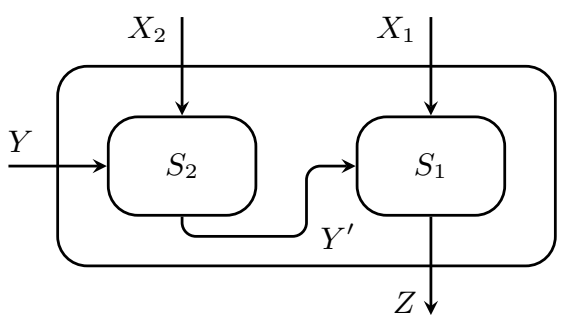

Figure 4: The operation $S_{1} \circ S_{2}$.

\subsection{Construction of the inner sampler}

We use the basic curve samplers as the building blocks and apply the error reduction as well as iterated sampling repeatedly to obtain the inner sampler. The formal construction is as follows. 
Definition 4.4 (inner sampler). For $m \geq 1, \delta>0$ and prime power $q$, pick $s=\lceil\log m\rceil$ and let $d_{i}=2^{s-i}$ for $0 \leq i \leq s$. Let $n_{i}=16^{i}$ for $0 \leq i \leq s-1$ and $n_{s}=16^{s}+20\left\lceil\log _{q}(1 / \delta)\right\rceil$. Define $S_{i}: \mathbb{F}_{q}^{n_{i} d_{i}} \times \mathbb{F}_{q}^{d_{i}} \rightarrow \mathbb{F}_{q}^{m}$ for $0 \leq i \leq s$ as follows:

- $S_{0}: \mathbb{F}_{q} \times \mathbb{F}_{q}^{d_{0}} \rightarrow \mathbb{F}_{q}^{m}$ projects $(x, y)$ onto the first $m$ coordinates of $y$.

- $S_{i} \stackrel{\text { def }}{=}\left(S_{i-1} \star \operatorname{RSCon} \frac{n_{i}}{4}, 2 n_{i-1}, q^{d_{i}}\right) \circ$ Curve $_{3, \frac{n_{i}}{4}, q^{d_{i}}}$ for $i=1, \ldots, s$.

Finally, let InnerSamp $m, \delta, q \stackrel{\text { def }}{=} S_{s}$.

See Figure 5 for an illustration of the above construction.

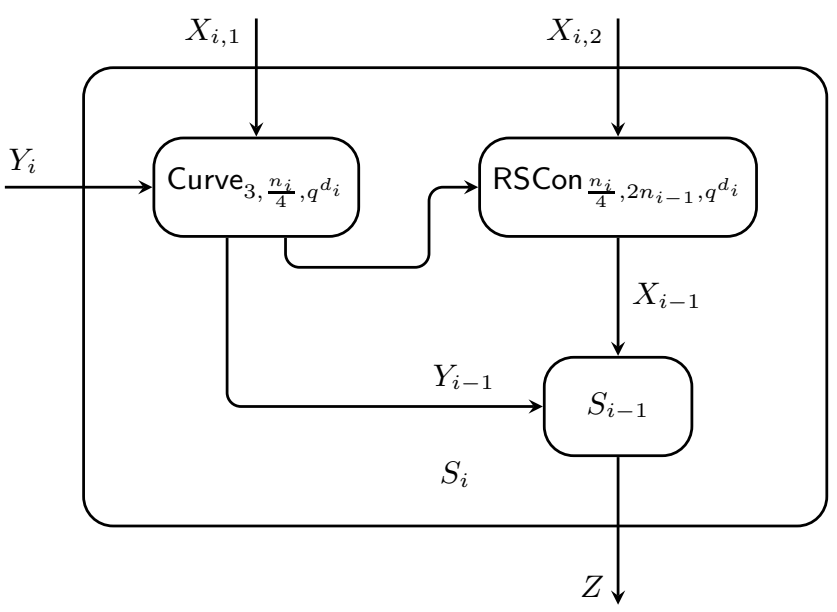

Figure 5: The recursive construction of $S_{i}=\left(S_{i-1} \star \operatorname{RSCon} \frac{n_{i}}{4}, 2 n_{i-1}, q^{d_{i}}\right) \circ \operatorname{Curve}_{3, \frac{n_{i}}{4}, q^{d_{i}}}$ used to define the inner sampler. Here $X_{i}=\left(X_{i, 1}, X_{i, 2}\right)$ (resp. $\left.X_{i-1}\right)$ and $Y_{i}$ (resp. $\left.Y_{i-1}\right)$ are the two arguments of $S_{i}$ (resp. $S_{i-1}$ ). And $Z$ is the common output of $S_{i}$ and $S_{i-1}$.

Theorem 4.2. For any $\epsilon, \delta>0$, integer $m \geq 1$ and large enough prime power $q \geq\left(\frac{m \log (1 / \delta)}{\epsilon}\right)^{O(1)}$, InnerSamp ${ }_{m, \delta, q}: \mathbb{F}_{q}^{n} \times \mathbb{F}_{q} \rightarrow \mathbb{F}_{q}^{m}$ is an $(\epsilon, \delta)$ sampler of degree $t$ where $n=O\left(m^{O(1)}+\log _{q}(1 / \delta)\right)$ and $t=O\left(m^{O(\log m)} \log _{q}^{2}(1 / \delta)\right)$.

Proof. Let $\epsilon^{\prime}=\frac{\epsilon}{2 s}$ and $d_{i}, n_{i}, S_{i}$ be as in Definition 4.4 for $0 \leq i \leq s$. We will prove that each $S_{i}$ is an $\left(\epsilon_{i}, \delta_{i}\right)$ sampler of degree $t_{i}$ where $\epsilon_{i}=2 i \epsilon^{\prime}, \delta_{i}=q^{-n_{i} d_{i} / 20}$, and $t_{i}=\prod_{j=1}^{i}\left(\frac{n_{j}}{4}\right)^{2}$. The theorem follows by noting that InnerSamp $m, \delta, q=S_{s}, \epsilon=\epsilon_{s}, \delta \geq \delta_{s}$, and $t=t_{s}$.

Induct on $i$. The case $i=0$ is trivial. Consider the case $i>0$ and assume the claim holds for all $i^{\prime}<i$. By the induction hypothesis, $S_{i-1}$ is an $\left(\epsilon_{i-1}, \delta_{i-1}\right)$ sampler of degree $t_{i-1}$.

Note that $\delta_{i-1}=q^{-n_{i-1} d_{i-1} / 20} \leq q^{-0.01 n_{i-1} d_{i-1}}$. By Corollary 4.1, $S_{i-1} \star \operatorname{RSCon} \frac{n_{i}, 2 n_{i-1}, q^{d_{i}}}{4}$ is an $\left(\epsilon_{i-1}+\epsilon^{\prime}, q^{n_{i-1} d_{i-1}-\left(n_{i} / 4\right) d_{i}}\right)$ sampler of degree $\frac{n_{i}}{4} \cdot t_{i-1}$. By Lemma 2.3, Curve $3, \frac{n_{i}}{4}, q^{d_{i}}$ is an $\left(\epsilon^{\prime}, q^{-n_{i} d_{i} / 16}\right)$ sampler of degree $\frac{n_{i}}{4}$. Finally by Lemma 4.2 , the function

$$
S_{i}=\left(S_{i-1} \star \operatorname{RSCon} \frac{n_{i}}{4}, 2 n_{i-1}, q^{d_{i}}\right) \circ \operatorname{Curve}_{3, \frac{n_{i}}{4}, q^{d_{i}}}
$$


is an $\left(\epsilon_{i-1}+2 \epsilon^{\prime}, q^{n_{i-1} d_{i-1}-\left(n_{i} / 4\right) d_{i}}+q^{-n_{i} d_{i} / 16}\right)$ sampler of degree $\left(\frac{n_{i}}{4}\right)^{2} \cdot t_{i-1}$. It remains to check that

$$
\epsilon_{i}=\epsilon_{i-1}+2 \epsilon^{\prime}, \quad \delta_{i} \geq q^{n_{i-1} d_{i-1}-\left(n_{i} / 4\right) d_{i}}+q^{-n_{i} d_{i} / 16} \quad \text { and } \quad t_{i}=\left(\frac{n_{i}}{4}\right)^{2} \cdot t_{i-1} .
$$

which hold by the choices of parameters.

\section{Putting it together}

We compose the outer sampler and the inner sampler to get the desired curve sampler.

Definition 5.1. For $m \geq 1, \delta>0$ and prime power $q$, define

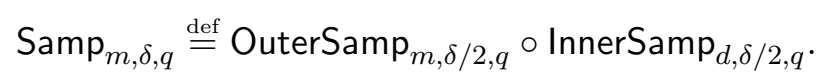

Theorem 5.1 (Theorem 1.1 restated). For any $\epsilon, \delta>0$, integer $m \geq 1$ and sufficiently large prime power $q \geq\left(\frac{m \log (1 / \delta)}{\epsilon}\right)^{O(1)}$, the function $\mathrm{Samp}_{m, \delta, q}: \mathbb{F}_{q}^{n} \times \mathbb{F}_{q} \rightarrow \mathbb{F}_{q}^{m}$ is an $(\epsilon, \delta)$ sampler of degree $t$ where $n=O\left(m+\log _{q}(1 / \delta)\right)$ and $t=\left(m \log _{q}(1 / \delta)\right)^{O(1)}$. In particular, Samp $\mathrm{S}_{m, \delta}$ is an $(\epsilon, \delta)$ degree-t curve sampler.

Proof. By Theorem 3.3, OuterSamp ${ }_{m, \delta / 2, q}: \mathbb{F}_{q}^{n_{1}} \times \mathbb{F}_{q}^{d} \rightarrow \mathbb{F}_{q}^{m}$ is an $(\epsilon / 2, \delta / 2)$ sampler of degree $t_{1}$ where $d=O(\log m), n_{1}=O\left(m+\log _{q}(1 / \delta)\right)$ and $t_{1}=O\left(m^{2}+m \log _{q}(1 / \delta)\right)$.

By Theorem 4.2, InnerSamp ${ }_{d, \delta / 2, q}: \mathbb{F}_{q}^{n_{2}} \times \mathbb{F}_{q} \rightarrow \mathbb{F}_{q}^{d}$ is an $(\epsilon / 2, \delta / 2)$ sampler of degree $t_{2}$ where $n_{2}=O\left((\log m)^{O(1)}+\log _{q}(1 / \delta)\right)$ and $t_{2}=O\left((\log m)^{O(\log \log m)} \log _{q}^{2}(1 / \delta)\right)$.

Finally, Lemma 4.2 implies that Samp $_{m, \delta, q}$ is an $(\epsilon, \delta)$ sampler of degree $t$ with $n=n_{1}+n_{2}=$ $O\left(m+\log _{q}(1 / \delta)\right)$ and $t=t_{1} t_{2}=\left(m \log _{q}(1 / \delta)\right)^{O(1)}$. A fortiori, it is a degree- $t$ curve sampler since the degree of $\operatorname{Samp}_{m, \delta, q}(x, \cdot)$ is bounded by the degree of Samp $m, \delta, q$ for all $x \in \mathbb{F}_{q}^{n}$.

\section{Acknowledgements}

The author is grateful to Chris Umans for his support and many helpful discussions.

\section{References}

[ALM $\left.{ }^{+} 98\right]$ Sanjeev Arora, Carsten Lund, Rajeev Motwani, Madhu Sudan, and Mario Szegedy. Proof verification and the hardness of approximation problems. J. ACM, 45(3):501555, May 1998.

[AS98] Sanjeev Arora and Shmuel Safra. Probabilistic checking of proofs: a new characterization of NP. J. ACM, 45(1):70-122, January 1998.

[BR94] M. Bellare and J. Rompel. Randomness-efficient oblivious sampling. In Proceedings of the 35th Annual Symposium on Foundations of Computer Science, SFCS '94, pages 276-287, Washington, DC, USA, 1994. IEEE Computer Society. 
[BSSVW03] Eli Ben-Sasson, Madhu Sudan, Salil Vadhan, and Avi Wigderson. Randomnessefficient low degree tests and short PCPs via epsilon-biased sets. In Proceedings of the 35th Annual ACM Symposium on Theory of Computing, STOC '03, pages 612621, New York, NY, USA, 2003. ACM.

[CG88] Benny Chor and Oded Goldreich. Unbiased bits from sources of weak randomness and probabilistic communication complexity. SIAM J. Comput., 17:230-261, April 1988.

[CG89] B. Chor and O. Goldreich. On the power of two-point based sampling. J. Complex., 5(1):96-106, April 1989.

[GI01] V. Guruswami and P. Indyk. Expander-based constructions of efficiently decodable codes. In Proceedings of the 42nd IEEE Symposium on Foundations of Computer Science, FOCS '01, pages 658-, Washington, DC, USA, 2001. IEEE Computer Society.

[Gil98] David Gillman. A Chernoff bound for random walks on expander graphs. SIAM J. Comput., 27(4):1203-1220, August 1998.

[Gol11] Oded Goldreich. A sample of samplers: A computational perspective on sampling. In Studies in Complexity and Cryptography, volume 6650 of Lecture Notes in Computer Science, pages 302-332. Springer Berlin Heidelberg, 2011.

[GR08] V. Guruswami and A. Rudra. Explicit codes achieving list decoding capacity: Errorcorrection with optimal redundancy. IEEE Trans. Inf. Theor., 54(1):135-150, January 2008 .

[GUV09] Venkatesan Guruswami, Christopher Umans, and Salil Vadhan. Unbalanced expanders and randomness extractors from Parvaresh-Vardy codes. J. ACM, 56:20:1-20:34, July 2009 .

[MR08] Dana Moshkovitz and Ran Raz. Sub-constant error low degree test of almost-linear size. SIAM J. Comput., 38(1):140-180, March 2008.

[NZ96] Noam Nisan and David Zuckerman. Randomness is linear in space. J. Comput. Syst. Sci., 52:43-52, February 1996.

[RSW06] Omer Reingold, Ronen Shaltiel, and Avi Wigderson. Extracting randomness via repeated condensing. SIAM J. Comput., 35(5):1185-1209, May 2006.

[RTS00] Jaikumar Radhakrishnan and Amnon Ta-Shma. Bounds for dispersers, extractors, and depth-two superconcentrators. SIAM J. Discret. Math., 13(1):2-24, January 2000.

[STV01] Madhu Sudan, Luca Trevisan, and Salil Vadhan. Pseudorandom generators without the XOR lemma. J. Comput. Syst. Sci., 62(2):236-266, March 2001.

[SU05] Ronen Shaltiel and Christopher Umans. Simple extractors for all min-entropies and a new pseudorandom generator. J. ACM, 52(2):172-216, March 2005.

[SU06] Ronen Shaltiel and Christopher Umans. Pseudorandomness for approximate counting and sampling. Comput. Complex., 15(4):298-341, December 2006. 
[SZ99] Aravind Srinivasan and David Zuckerman. Computing with very weak random sources. SIAM J. Comput., 28:1433-1459, March 1999.

[TSU06] Amnon Ta-Shma and Christopher Umans. Better lossless condensers through derandomized curve samplers. In Proceedings of the 47th Annual IEEE Symposium on Foundations of Computer Science, pages 177-186, Washington, DC, USA, 2006. IEEE Computer Society.

[TSU12] Amnon Ta-Shma and Christopher Umans. Better condensers and new extractors from parvaresh-vardy codes. In Proceedings of the 27th IEEE Conference on Computational Complexity, 2012.

[TSUZ07] Amnon Ta-Shma, Christopher Umans, and David Zuckerman. Lossless condensers, unbalanced expanders, and extractors. Combinatorica, 27(2):213-240, March 2007.

[Uma03] Christopher Umans. Pseudo-random generators for all hardnesses. J. Comput. Syst. Sci., 67(2):419-440, September 2003.

[Zuc97] David Zuckerman. Randomness-optimal oblivious sampling. In Proceedings of the Workshop on Randomized Algorithms and Computation, pages 345-367, New York, NY, USA, 1997. John Wiley \& Sons, Inc.

\section{A Tail probability bounds}

The following bound follows from Chebyshev's inequality:

Lemma A.1. Suppose $X_{1}, \ldots, X_{n}$ are pairwise independent random variables. Let $X=\sum_{i=1}^{n} X_{i}$ and $\mu=\mathbb{E}[X]$, and let $A>0$. Then

$$
\operatorname{Pr}[|X-\mu| \geq A] \leq \frac{\sum_{i=1}^{n} \operatorname{Var}\left[X_{i}\right]}{A^{2}} .
$$

We will also use the following tail bound for $t$-wise independent random variables:

Lemma A.2 ([BR94]). Let $t \geq 4$ be an even integer. Suppose $X_{1}, \ldots, X_{n}$ are $t$-wise independent random variables over $[0,1]$. Let $X=\sum_{i=1}^{n} X_{i}$ and $\mu=\mathbb{E}[X]$, and let $A>0$. Then

$$
\operatorname{Pr}[|X-\mu| \geq A]=O\left(\left(\frac{t \mu+t^{2}}{A^{2}}\right)^{t / 2}\right) .
$$

\section{B Lower bounds}

We will use the following optimal lower bound for extractors:

Theorem B.1 ([RTS00], restated). Let $E: \mathbb{F}_{q}^{n} \times \mathbb{F}_{q}^{d} \rightarrow \mathbb{F}_{q}^{m}$ be a $(k, \epsilon, q)$ extractor. Then

(a) if $\epsilon<1 / 2$ and $q^{d} \leq q^{m} / 2$, then $q^{d}=\Omega\left(\frac{(n-k) \log q}{\epsilon^{2}}\right)$, and

(b) if $q^{d} \leq q^{m} / 4$, then $q^{d+k-m}=\Omega\left(1 / \epsilon^{2}\right)$. 
Theorem B.2. Let $S: \mathbb{F}_{q}^{n} \times \mathbb{F}_{q} \rightarrow \mathbb{F}_{q}^{m}$ be an $(\epsilon, \delta)$ curve sampler where $\epsilon<1 / 2$ and $m \geq 2$. Then

(a) the sample complexity $q=\Omega\left(\frac{\log (2 \epsilon / \delta)}{\epsilon^{2}}\right)$, and

(b) the randomness complexity $n \log q \geq(m-1) \log q+\log (1 / \epsilon)+\log (1 / \delta)-O(1)$.

Proof. By Theorem 2.1, $S$ is a $(k, 2 \epsilon, q)$ extractor where $k=n-\log _{q}(2 \epsilon / \delta)$. The first claim then follows from Theorem B.1 (a). Applying Theorem B.1 (b), we get $(1+k-m) \log q \geq \Omega(\log (1 / \epsilon))-$ $O(1)$. Therefore

$$
n \log q=k \log q+\log (2 \epsilon / \delta) \geq(m-1) \log q+\log (1 / \delta)+\Omega(\log (1 / \epsilon))-O(1) .
$$

In particular, as $\log (1 / \epsilon)=O(\log q)$, the randomness complexity $n \log q$ is at least $\Omega(\log N+$ $\log (1 / \delta)$ ) when the domain size $N=q^{m} \geq N_{0}$ for some constant $N_{0}$. Therefore the randomness complexity in Theorem 1.1 is optimal up to a constant factor.

We also present the following lower bound on the degree of curves sampled by a curve sampler:

Theorem B.3. Let $S: \mathcal{N} \times \mathbb{F}_{q} \rightarrow \mathbb{F}_{q}^{m}$ be an $(\epsilon, \delta)$ degree-t curve sampler where $m \geq 2, \epsilon<1 / 2$ and $\delta<1$. Then $t=\Omega\left(\log _{q}(1 / \delta)+1\right)$.

Proof. Clearly $t \geq 1$. Suppose $S=\left(S_{1}, \ldots, S_{m}\right)$ and define $S^{\prime}=\left(S_{1}, S_{2}\right)$. Let $\mathcal{C}$ be the set of curves of degree at most $t$ in $\mathbb{F}_{q}^{2}$. Then $|\mathcal{C}|=q^{2(t+1)}$. Consider the map $\tau: \mathcal{N} \rightarrow \mathcal{C}$ that sends $x$ to $S^{\prime}(x, \cdot)$. We can pick $k=\lfloor q / 2\rfloor$ curves $C_{1}, \ldots, C_{k} \in \mathcal{C}$ such that the union of their preimages

$$
B \stackrel{\text { def }}{=} \bigcup_{i=1}^{k} \tau^{-1}\left(C_{i}\right)=\bigcup_{i=1}^{k}\left\{x: S^{\prime}(x, \cdot)=C_{i}\right\}
$$

has size at least $\frac{k|\mathcal{N}|}{|\mathcal{C}|}=\frac{k|\mathcal{N}|}{q^{2(t+1)}}$.

Define $A \subseteq \mathbb{F}_{q}^{m}$ by

$$
A \stackrel{\text { def }}{=}\left\{C_{i}(y): i \in[k], y \in \mathbb{F}_{q}\right\} \times \mathbb{F}_{q}^{m-2},
$$

i.e., let $A$ be the set of points in $\mathbb{F}_{q}^{m}$ whose first two coordinates are on at least one curve $C_{i}$. We have $|A| \leq k q^{m-1}$ and hence $\mu(A) \leq k / q \leq 1 / 2<1-\epsilon$. On the other hand, it follows from the definition of $A$ that we have $S(x, y) \in A$ for all $x \in B$ and $y \in \mathbb{F}_{q}$. So $\mu_{S(x)}(A)=1$ for all $x \in B$. Then $\delta \geq \operatorname{Pr}\left[\left|\mu_{S(x)}(A)-\mu(A)\right|>\epsilon\right] \geq \frac{|B|}{|\mathcal{N}|} \geq \frac{k}{q^{2(t+1)}}$ and hence $t \geq \max \left\{1, \frac{1}{2} \log _{q}(k / \delta)-1\right\}=$ $\Omega\left(\log _{q}(1 / \delta)+1\right)$.

We remark that the condition $m \geq 2$ is necessary in Theorem B.3 since when $m=1$, the sampler $S$ with $S(x, y)=y$ for all $x \in \mathcal{N}$ and $y \in \mathbb{F}_{q}$ is a $(0,0)$ degree-1 curve sampler.

\section{Omitted Proofs}

Lemma 2.1 (from page 5). A manifold $f:\left(\mathbb{F}_{q^{D}}\right)^{n} \rightarrow\left(\mathbb{F}_{q^{D}}\right)^{m}$ of degree $t$, when viewed as a manifold $f:\left(\mathbb{F}_{q}^{D}\right)^{n} \rightarrow\left(\mathbb{F}_{q}^{D}\right)^{m}$, also has degree at most $t$. 
Proof. Write $f=\left(f_{1}, \ldots, f_{m}\right)$. By symmetry we just show $\operatorname{deg}\left(f_{1}\right) \leq t$ as a polynomial map over $\mathbb{F}_{q}$. Suppose

$$
f_{1}\left(x_{1}, \ldots, x_{n}\right)=\sum_{\substack{d=\left(d_{1}, \ldots, d_{n}\right) \\ \sum d_{i} \leq t}} c_{d} \prod_{i=1}^{n} x_{i}^{d_{i}} .
$$

Let $\left(e_{1}, \ldots, e_{D}\right)$ be the standard basis of $\mathbb{F}_{q}^{D}$ over $\mathbb{F}_{q}$. Writing the $i$-th variable $x_{i} \in \mathbb{F}_{q D}$ as $\sum_{j=1}^{D} x_{i, j} e_{j} \in \mathbb{F}_{q}^{D}$ with $x_{i, j} \in \mathbb{F}_{q}$, and each coefficient $c_{d} \in F_{q D}$ as $\sum_{j=1}^{D} c_{d, j} e_{j}$ with $c_{d, j} \in \mathbb{F}_{q}$, we obtain

$$
f_{1}\left(x_{1}, \ldots, x_{n}\right)=\sum_{\substack{d=\left(d_{1}, \ldots, d_{n}\right) \\ \sum d_{i} \leq t}}\left(\sum_{j=1}^{D} c_{d, j} e_{j}\right) \prod_{i=1}^{n}\left(\sum_{j=1}^{D} x_{i, j} e_{j}\right)^{d_{i}} .
$$

After multiplying out, each monomial has degree $\max _{d} \sum_{i} d_{i} \leq t$, and their coefficients are polynomials in the $e_{i}$ elements. Rewriting each of these values in the basis $\left(e_{1}, \ldots, e_{D}\right)$ and gathering the coefficients on $e_{i}$, we obtain the $i$-th coordinate function of $f_{1}$ that has degree at most $t$ for all $1 \leq i \leq D$. Therefore $f_{1}:\left(\mathbb{F}_{q}^{D}\right)^{n} \rightarrow \mathbb{F}_{q}^{D}$ is a manifold of degree at most $t$, and so is $f:\left(\mathbb{F}_{q}^{D}\right)^{n} \rightarrow\left(\mathbb{F}_{q}^{D}\right)^{m}$.

Lemma 2.2 (from page 6). For $\epsilon>0, m \geq 1$ and prime power $q$, Line L,$q$ is an $\left(\epsilon, \frac{1}{\epsilon^{2} q}\right)$ line sampler.

Proof. Let $A$ be an arbitrary subset of $\mathbb{F}_{q}^{m}$. Note that Line $m, q$ picks a line uniformly at random. It is well known that the random variables Line $\operatorname{Lin}_{q}\left(U_{2 m, q}, y\right)$ with $y$ ranging over $\mathbb{F}_{q}$ are pairwise independent. So the indicator variables $\mathbf{I}\left[\right.$ Line $\left._{m, q}\left(U_{2 m, q}, y\right) \in A\right]$ with $y$ ranging over $\mathbb{F}_{q}$ are also pairwise independent. Applying Lemma A.1, we get

$$
\begin{aligned}
& \operatorname{Pr}_{x \leftarrow U_{2 m, q}}\left[\left|\mu_{\operatorname{Line}_{m, q}(x)}(A)-\mu(A)\right|>\epsilon\right] \\
= & \operatorname{Pr}\left[\mid \sum_{y \in \mathbb{F}_{q}} \mathbf{I}\left[\operatorname{Line}_{m, q}\left(U_{2 m, q}, y\right) \in A\right]-\mathbb{E}\left[\sum_{y \in \mathbb{F}_{q}} \mathbf{I}\left[\text { Line }_{m, q}\left(U_{2 m, q}, y\right) \in A\right]\right] \mid>\epsilon q\right] \\
\leq & \frac{\sum_{y \in \mathbb{F}_{q}} \operatorname{Var}\left[\mathbf{I}\left[\operatorname{Line}_{m, q}\left(U_{2 m, q}, y\right) \in A\right]\right]}{\epsilon^{2} q^{2}} \\
\leq & \frac{1}{\epsilon^{2} q}
\end{aligned}
$$

By definition, Line L $_{q}$ is an $\left(\epsilon, \frac{1}{\epsilon^{2} q}\right)$ line sampler.

Lemma 2.3 (from page 6). For $\epsilon>0, m \geq 1, t \geq 4$ and sufficiently large prime power $q=$ $(t / \epsilon)^{O(1)}$, Curve m,t,q $_{\text {is }}$ an $\left(\epsilon, q^{-t / 4}\right)$ sampler.

Proof. Let $A$ be an arbitrary subset of $\mathbb{F}_{q}^{m}$. Note that Curve ${ }_{m, t, q}$ picks a degree- $(t-1)$ curve uniformly at random. It is well known that the random variables Curve $_{m, t, q}\left(U_{t m, q}, y\right)$ with $y$ ranging 
over $\mathbb{F}_{q}$ are $t$-wise independent. So the indicator variables $\mathbf{I}\left[\right.$ Curve $\left._{m, t, q}\left(U_{t m, q}, y\right) \in A\right]$ with $y$ ranging over $\mathbb{F}_{q}$ are also $t$-wise independent. Applying Lemma A.2, we get

$$
\begin{aligned}
& \operatorname{Pr}_{x \leftarrow U U_{t m, q}}\left[\left|\mu_{\text {Curve }_{m, t, q}(x)}(A)-\mu(A)\right|>\epsilon\right] \\
& =\operatorname{Pr}\left[\mid \sum_{y \in \mathbb{F}_{q}} \mathbf{I}\left[\text { Curve }_{m, t, q}\left(U_{t m, q}, y\right) \in A\right]-\mathbb{E}\left[\sum_{y \in \mathbb{F}_{q}} \mathbf{I}\left[\text { Curve }_{m, t, q}\left(U_{t m, q}, y\right) \in A\right]\right]>\epsilon q\right] \\
& =O\left(\left(\frac{t q \mu(A)+t^{2}}{\epsilon^{2} q^{2}}\right)^{t / 2}\right) \leq q^{-t / 4}
\end{aligned}
$$

provided that $q \geq(t / \epsilon)^{O(1)}$ is sufficiently large. By definition, Curve $e_{m, t, q}$ is an $\left(\epsilon, q^{-t / 4}\right)$ sampler.

Theorem 2.1 (from page 6). Given a map $f: \mathbb{F}_{q}^{n} \times \mathbb{F}_{q}^{d} \rightarrow \mathbb{F}_{q}^{m}$, we have the following:

1. If $f$ is a $(k, \epsilon, q)$ extractor, then it is also an $(\epsilon, \delta)$ sampler where $\delta=2 q^{k-n}$.

2. If $f$ is an $(\epsilon / 2, \delta)$ sampler where $\delta=\epsilon q^{k-n}$, then it is also a $(k, \epsilon, q)$ extractor.

Proof. (Extractor to sampler) Assume to the contrary that $f$ is not an $(\epsilon, \delta)$ sampler. Then there exists a subset $A \subseteq \mathbb{F}_{q}^{m}$ with $\operatorname{Pr}_{x}\left[\left|\mu_{f(x)}(A)-\mu(A)\right|>\epsilon\right]>\delta$. Then either $\operatorname{Pr}_{x}\left[\mu_{f(x)}(A)-\mu(A)>\epsilon\right]>$ $\delta / 2$ or $\operatorname{Pr}_{x}\left[\mu_{f(x)}(A)-\mu(A)<-\epsilon\right]>\delta / 2$. Assume $\operatorname{Pr}_{x}\left[\mu_{f(x)}(A)-\mu(A)>\epsilon\right]>\delta / 2$ (the other case is symmetric). Let $X$ be the uniform distribution over the set of $x$ such that $\mu_{f(x)}(A)-\mu(A)>\epsilon$, i.e., $\operatorname{Pr}_{y}[f(x, y) \in A]-\operatorname{Pr}_{x}[x \in A]>\epsilon$. Then $\left|f\left(X, U_{d, q}\right)-U_{m, q}\right|>\epsilon$. But the $q$-ary min-entropy of $X$ is at least $\log _{q}\left((\delta / 2) q^{n}\right) \geq k$, contradicting the extractor property of $f$.

(Sampler to extractor) Assume to the contrary that $f$ is not a $(k, \epsilon, q)$ extractor. Then there exists a subset $A \subseteq \mathbb{F}_{q}^{m}$ and a random source $X$ of $q$-ary min-entropy $k$ satisfying the property that $\left|\operatorname{Pr}\left[f\left(X, U_{d, q}\right) \in A\right]-\mu(A)\right|>\epsilon$. We may assume $X$ is a flat source (i.e. uniformly distributed over its support) since a general source with $q$-ary min-entropy $k$ is a convex combination of flat sources with $q$-ary min-entropy $k$. Note that $|\operatorname{supp}(X)| \geq q^{k}$. By the averaging argument, for at least an $\epsilon$-fraction of $x \in \operatorname{supp}(X)$, we have $\left|\operatorname{Pr}\left[f\left(x, U_{d, q}\right) \in A\right]-\mu(A)\right|>\epsilon / 2$. But it implies that for $x$ uniformly chosen from $\mathbb{F}_{q}^{n}$, with probability at least $\epsilon \mu(\operatorname{supp}(X))=\delta$ we have $\left|\mu_{f(x)}(A)-\mu(A)\right|>\epsilon / 2$, contradicting the sampling property of $f$.

Lemma 3.1 (from page 7). Let $s \geq 1$ be an integer and for each $i \in[s]$, let $E_{i}: \mathbb{F}_{q}^{n_{i}} \times \mathbb{F}_{q}^{d_{i}} \rightarrow \mathbb{F}_{q}^{m_{i}}$ be $a\left(k_{i}, \epsilon_{i}, q\right)$ extractor of degree $t_{i} \geq 1$. Then $\operatorname{BlkExt}\left(E_{1}, \ldots, E_{s}\right)$ is a $\left(\left(k_{1}, \ldots, k_{s}\right), \epsilon, q\right)$ block source extractor of degree $t$ where $\epsilon=\sum_{i=1}^{s} \epsilon_{i}$ and $t=\prod_{i=1}^{s} t_{i}$.

Proof. Induct on $s$. When $s=1$ the claim follows from the extractor property of $E_{1}$.

When $s>1$, assume the claim holds for all $s^{\prime}<s$. Let $E=\operatorname{BlkExt}\left(E_{1}, \ldots, E_{s}\right)$ and $E^{\prime}=$ $\operatorname{BlkExt}\left(E_{2}, \ldots, E_{s}\right)$. By the induction hypothesis, $E^{\prime}$ is a $\left(\left(k_{2}, \ldots, k_{s}\right), \epsilon^{\prime}, q\right)$ block source extractor of degree $t^{\prime}$ where $\epsilon^{\prime}=\sum_{i=2}^{s} \epsilon_{i}$ and $t^{\prime}=\prod_{i=2}^{s} t_{i}$. Let $\left(X_{1}, \ldots, X_{s}\right)$ be an arbitrary $\left(k_{1}, \ldots, k_{s}\right)$ $q$-ary block source over $\mathbb{F}_{q}^{n_{1}} \times \cdots \times \mathbb{F}_{q}^{n_{s}}$. Let $\left(Y_{i-1}, Z_{i}\right)$ be the output of $E_{i}$ and $\left(Z_{1}, \ldots, Z_{s}\right)$ be the output of $E$ when $\left(X_{1}, \ldots, X_{s}\right)$ is fed to $E$ as the input and an independent uniform distribution $Y_{s}=U_{d_{s}, q}$ is used as the seed. The output of $E^{\prime}$ is then $\left(Y_{1}, Z_{2}, \ldots, Z_{s}\right)$. 
Fix $x \in \operatorname{supp}\left(X_{1}\right)$. By the definition of block sources, the distribution $\left.\left(X_{2}, \ldots, X_{s}\right)\right|_{X_{1}=x}$ is a $\left(k_{2}, \ldots, k_{s}\right) q$-ary block source. Also note that $\left.Y_{s}\right|_{X_{1}=s}=Y_{s}$ is uniform distributed and independent from $X_{2}, \ldots, X_{s}$. By the induction hypothesis, the distribution

$$
\left.\left(Y_{1}, Z_{2}, \ldots, Z_{s}\right)\right|_{X_{1}=x}=\left.E^{\prime}\left(\left(X_{2}, \ldots, X_{s}\right), Y_{s}\right)\right|_{X_{1}=x}
$$

is $\epsilon^{\prime}$-close to $\left(U_{d_{1}, q}, U_{m_{2}-d_{1}, q}, \ldots, U_{m_{s}-d_{s-1}, q}\right)$. As this holds for all $x \in \operatorname{supp}\left(X_{1}\right)$, the distribution $\left(X_{1}, Y_{1}, Z_{2}, \ldots, Z_{s}\right)$ is $\epsilon^{\prime}$-close to $\left(X_{1}, U_{d_{1}, q}, U_{m_{2}-d_{1}, q}, \ldots, U_{m_{s}-d_{s-1}, q}\right)$. So the distribution

$$
E\left(\left(X_{1}, \ldots, X_{s}\right), Y_{s}\right)=\left(E_{1}\left(X_{1}, Y_{1}\right), Z_{2}, \ldots, Z_{s}\right)
$$

is $\epsilon^{\prime}$-close to $\left(E_{1}\left(X_{1}, U_{d_{1}, q}\right), U_{m_{2}-d_{1}, q}, \ldots, U_{m_{s}-d_{s-1}, q}\right)$. Then we know that it is also $\epsilon$-close to $\left(U_{m_{1}-d_{0}, q}, U_{m_{2}-d_{1}, q}, \ldots, U_{m_{s}-d_{s-1}, q}\right)$ since $E_{1}$ is a $\left(k_{1}, \epsilon_{1}, q\right)$ extractor.

Finally, to see that $E$ has degree $t$, note that $E^{\prime}\left(\left(X_{2}, \ldots, X_{s}\right), Y_{s}\right)=\left(Y_{1}, Z_{2}, \ldots, Z_{s}\right)$ has degree $t^{\prime}$ in its variables $X_{2}, \ldots, X_{s}$ and $Y_{s}$ by the induction hypothesis and hence $Y_{1}, Z_{2}, \ldots, Z_{s}$ have degree $t^{\prime}$ in these variables. Then $Z_{1}=E_{1}\left(X_{1}, Y_{1}\right)$ has degree $t_{1} \cdot \max \left\{1, t^{\prime}\right\}=t$ in $X_{1}, \ldots, X_{s}$ and $Y_{s}$. So $E\left(\left(X_{1}, \ldots, X_{s}\right), Y_{s}\right)=\left(Z_{1}, \ldots, Z_{s}\right)$ has degree $t$ in $X_{1}, \ldots, X_{s}$ and $Y_{s}$.

Theorem 3.2 (from page 8). For $\epsilon>0$, integers $s, n, m_{1}, \ldots, m_{s} \geq 1$ and sufficiently large prime power $q=(n / \epsilon)^{O(1)}$, the function $\mathrm{BlkCnvt}_{n,\left(m_{1}, \ldots, m_{s}\right), q}$ is a $\left(k,\left(k_{1}, \ldots, k_{s}\right), 3 s \epsilon, q\right)$ block source converter of degree $n$ where $k=\sum_{i=1}^{s} m_{i}+\log _{q}(1 / \epsilon)$ and each $k_{i}=0.99 m_{i}$.

We first prove the following technical lemmas.

Lemma C.1 (chain rule for min-entropy). Let $(X, Y)$ be a joint distribution where $X$ is distributed over $\mathbb{F}_{q}^{\ell}$ and $Y$ has q-ary min-entropy $k$. We have

$$
\operatorname{Pr}_{x \leftarrow X}\left[\left.Y\right|_{X=x} \text { has q-ary min-entropy } k-\ell-\log _{q}(1 / \epsilon)\right] \geq 1-\epsilon .
$$

Proof. We say $x \in \operatorname{supp}(X)$ is good if $\operatorname{Pr}[X=x] \geq \epsilon q^{-\ell}$ and bad otherwise. Then $\operatorname{Pr}_{x \leftarrow X}[x$ is bad $] \leq$ $\operatorname{supp}(X) \epsilon q^{-\ell} \leq \epsilon$. Consider arbitrary good $x$. For any specific value $y$ for $Y$, we have $\operatorname{Pr}\left[\left.Y\right|_{X=x}=\right.$ $y]=\frac{\operatorname{Pr}[(Y=y) \wedge(X=x)]}{\operatorname{Pr}[X=x]} \leq \frac{\operatorname{Pr}[Y=y]}{\epsilon q^{-\ell}} \leq q^{-\left(k-\ell-\log _{q}(1 / \epsilon)\right)}$. By definition, $\left.Y\right|_{X=x}$ has $q$-ary min-entropy $k-\ell-\log _{q}(1 / \epsilon)$ when $X=x$ is good, which occurs with probability at least $1-\epsilon$.

Lemma C.2. Let $P, Q$ be two distributions over set $I$ with $\Delta(P, Q) \leq \epsilon$. Let $\left\{X_{i}: i \in \operatorname{supp}(P)\right\}$ and $\left\{Y_{i}: i \in \operatorname{supp}(Q)\right\}$ be two collections of distributions over the same set $S$ such that $\Delta\left(X_{i}, Y_{i}\right) \leq$ $\epsilon^{\prime}$ for any $i \in \operatorname{supp}(P) \cap \operatorname{supp}(Q)$. Then $X \stackrel{\text { def }}{=} \sum_{i \in \operatorname{supp}(P)} \operatorname{Pr}[P=i] \cdot X_{i}$ is $\left(2 \epsilon+\epsilon^{\prime}\right)$-close to $Y \stackrel{\text { def }}{=} \sum_{i \in \operatorname{supp}(Q)} \operatorname{Pr}[Q=i] \cdot Y_{i}$.

Proof. Let $T$ be an arbitrary subset of $S$ and we will prove that $|\operatorname{Pr}[X \in T]-\operatorname{Pr}[Y \in T]| \leq 2 \epsilon+\epsilon^{\prime}$.

Note that we can add dummy distributions $X_{i}$ for $i \in I \backslash \operatorname{supp}(P)$ and $Y_{j}$ for $j \in I \backslash \operatorname{supp}(Q)$ such that $\Delta\left(X_{i}, Y_{i}\right) \leq \epsilon^{\prime}$ for all $i \in I$, and it still holds that $X=\sum_{i \in I} \operatorname{Pr}[P=i] \cdot X_{i}$ and 
$Y=\sum_{i \in I} \operatorname{Pr}[Q=i] \cdot Y_{i}$. Then we have

$$
\begin{aligned}
& |\operatorname{Pr}[X \in T]-\operatorname{Pr}[Y \in T]| \\
& =\left|\sum_{i \in I} \operatorname{Pr}[P=i] \operatorname{Pr}\left[X_{i} \in T\right]-\sum_{i \in I} \operatorname{Pr}[Q=i] \operatorname{Pr}\left[Y_{i} \in T\right]\right| \\
& \leq \sum_{i \in I}\left|\operatorname{Pr}[P=i] \operatorname{Pr}\left[X_{i} \in T\right]-\operatorname{Pr}[Q=i] \operatorname{Pr}\left[Y_{i} \in T\right]\right| \\
& \leq \sum_{i \in I}\left|(\operatorname{Pr}[P=i]-\operatorname{Pr}[Q=i]) \operatorname{Pr}\left[X_{i} \in T\right]+\operatorname{Pr}[Q=i]\left(\operatorname{Pr}\left[X_{i} \in T\right]-\operatorname{Pr}\left[Y_{i} \in T\right]\right)\right| \\
& \leq\left(\sum_{i \in I}|\operatorname{Pr}[P=i]-\operatorname{Pr}[Q=i]|\right)+\epsilon^{\prime}\left(\sum_{i \in I} \operatorname{Pr}[Q=i]\right) \\
& \leq 2 \epsilon+\epsilon^{\prime} .
\end{aligned}
$$

Lemma C.3. Let $X=\left(X_{1}, \ldots, X_{s}\right)$ be a distribution over $\mathbb{F}_{q}^{n_{1}} \times \cdots \times \mathbb{F}_{q}^{n_{s}}$ such that for any $i \in[s]$ and $\left(x_{1}, \ldots, x_{i-1}\right) \in \operatorname{supp}\left(X_{1}, \ldots, X_{i-1}\right)$, the conditional distribution $\left.X_{i}\right|_{X_{1}=x_{1}, \ldots, X_{i-1}=x_{i-1}}$ is $\epsilon$-close to a distribution $\tilde{X}_{i}\left(x_{1}, \ldots, x_{i-1}\right)$ with q-ary min-entropy $k_{i}$. Then $X$ is $2 s \epsilon$-close to a $\left(k_{1}, \ldots, k_{s}\right)$ q-ary block source.

Proof. Define $X^{\prime}=\left(X_{1}^{\prime}, \ldots, X_{s}^{\prime}\right)$ as the unique distribution such that for any $i \in[s]$ and any $\left(x_{1}, \ldots, x_{i-1}\right) \in \operatorname{supp}\left(X_{1}^{\prime}, \ldots, X_{i-1}^{\prime}\right)$, the conditional distribution $\left.X_{i}^{\prime}\right|_{X_{1}^{\prime}=x_{1}, \ldots, X_{i-1}^{\prime}=x_{i-1}}$ equals the distribution $\tilde{X}_{i}\left(x_{1}, \ldots, x_{i-1}\right)$ if $\left(x_{1}, \ldots, x_{i-1}\right) \in \operatorname{supp}\left(X_{1}, \ldots, X_{i-1}\right)^{1}$ and otherwise equals $U_{n_{i}, q}$.

For any $i \in[s]$ and $\left(x_{1}, \ldots, x_{i-1}\right) \in \operatorname{supp}\left(X_{1}^{\prime}, \ldots, X_{i-1}^{\prime}\right)$, we known $\left.X_{i}^{\prime}\right|_{X_{1}^{\prime}=x_{1}, \ldots, X_{i-1}^{\prime}=x_{i-1}}$ is either $\tilde{X}_{i}\left(x_{1}, \ldots, x_{i-1}\right)$ or $U_{n_{i}, q}$. And in either case it has $q$-ary min-entropy $k_{i}$. So $X^{\prime}$ is a $\left(k_{1}, \ldots, k_{s}\right)$ $q$-ary block source.

We then prove that for any $i \in[s]$ and $\left(x_{1}, \ldots, x_{i-1}\right) \in \operatorname{supp}\left(X_{1}, \ldots, X_{i-1}\right) \cap \operatorname{supp}\left(X_{1}^{\prime}, \ldots, X_{i-1}^{\prime}\right)$, the conditional distribution $\left.X\right|_{X_{1}=x_{1}, \ldots, X_{i-1}=x_{i-1}}$ is $2(s-i+1) \epsilon$-close to $\left.X^{\prime}\right|_{X_{1}^{\prime}=x_{1}, \ldots, X_{i-1}^{\prime}=x_{i-1}}$. Setting $i=1$ proves the lemma.

Induct on $i$. For $i=s$ the claim holds by the definition of $X^{\prime}$. For $i<s$, assume the claim holds for $i+1$ and we prove that it holds for $i$ as well. Consider any $\left(x_{1}, \ldots, x_{i-1}\right) \in \operatorname{supp}\left(X_{1}, \ldots, X_{i-1}\right) \cap$ $\operatorname{supp}\left(X_{1}^{\prime}, \ldots, X_{i-1}^{\prime}\right)$. Let $A=\left.X_{i}\right|_{X_{1}=x_{1}, \ldots, X_{i-1}=x_{i-1}}$ and $B=\left.X_{i}^{\prime}\right|_{X_{1}^{\prime}=x_{1}, \ldots, X_{i-1}^{\prime}=x_{i-1}}$. We have

$$
\left.X\right|_{X_{1}=x_{1}, \ldots, X_{i-1}=x_{i-1}}=\left.\sum_{x_{i} \in \operatorname{supp}(A)} \operatorname{Pr}\left[A=x_{i}\right] \cdot X\right|_{X_{1}=x_{1}, \ldots, X_{i}=x_{i}}
$$

and

$$
\left.X^{\prime}\right|_{X_{1}^{\prime}=x_{1}, \ldots, X_{i-1}^{\prime}=x_{i-1}}=\left.\sum_{x_{i} \in \operatorname{supp}(B)} \operatorname{Pr}\left[B=x_{i}\right] \cdot X^{\prime}\right|_{X_{1}^{\prime}=x_{1}, \ldots, X_{i}^{\prime}=x_{i}} .
$$

By the induction hypothesis, we have

$$
\Delta\left(\left.X\right|_{X_{1}=x_{1}, \ldots, X_{i}=x_{i}},\left.X^{\prime}\right|_{X_{1}^{\prime}=x_{1}, \ldots, X_{i}^{\prime}=x_{i}}\right) \leq 2(s-i) \epsilon
$$

\footnotetext{
${ }^{1}\left(x_{1}, \ldots, x_{i-1}\right) \in \operatorname{supp}\left(X_{1}, \ldots, X_{i-1}\right)$ always holds if $i=1$.
} 
for $x_{i} \in \operatorname{supp}(A) \cap \operatorname{supp}(B)$. Also note that $B$ is identical to $\tilde{X}_{i}\left(x_{1}, \ldots, x_{i-1}\right)$ and is $\epsilon$-close to $A$. The claim then follows from Lemma C.2.

Now we are ready to prove Theorem 3.2.

Proof of Theorem 3.2. The degree of $\mathrm{BlkCnvt}_{n,\left(m_{1}, \ldots, m_{s}\right), q}$ is $n$ since $\mathrm{RSCon}_{n, m, q}$ has degree $n$. Let $X$ be a random source that has $q$-ary min-entropy $k$. Let $Y_{1}, \ldots, Y_{s}$ be independent seeds uniformly distributed over $\mathbb{F}_{q}$. Let $Z=\left(Z_{1}, \ldots, Z_{s}\right)=\operatorname{BlkCnvt}_{n,\left(m_{1}, \ldots, m_{s}\right), q}\left(X,\left(Y_{1}, \ldots, Y_{s}\right)\right)$ where each $Z_{i}=$ $\operatorname{RSCon}_{n, m_{i}, q}\left(X, Y_{i}\right)$ is distributed over $\mathbb{F}_{q}^{m_{i}}$. Define

$$
B=\left\{\left(z_{1}, \ldots, z_{i}\right): \begin{array}{l}
i \in[s],\left(z_{1}, \ldots, z_{i}\right) \in \operatorname{supp}\left(Z_{1}, \ldots, Z_{i}\right),\left.X\right|_{Z_{1}=z_{1}, \ldots, Z_{i}=z_{i}} \text { does not } \\
\text { have } q \text {-ary min-entropy } k-\left(m_{1}+\cdots+m_{i}\right)-\log _{q}(1 / \epsilon)
\end{array}\right\} .
$$

Define a new distribution $Z^{\prime}=\left(Z_{1}^{\prime}, \ldots, Z_{s}^{\prime}\right)$ as follows: Sample $z=\left(z_{1}, \ldots, z_{s}\right) \leftarrow Z$ and independently $u=\left(u_{1}, \ldots, u_{s}\right) \leftarrow U_{m_{1}+\ldots,+m_{s}, q}$. If there exist $i \in[s]$ such that $\left(z_{1}, \ldots, z_{i-1}\right) \in B$, then pick the smallest such $i$ and let $z^{\prime}=\left(z_{1}, \ldots, z_{i-1}, u_{i}, \ldots, u_{s}\right)$. Otherwise let $z^{\prime}=z$. Let $Z^{\prime}$ be the distribution of $z^{\prime}$.

For any $i \in[s]$ and $\left(z_{1}, \ldots, z_{i-1}\right) \in \operatorname{supp}\left(Z_{1}^{\prime}, \ldots, Z_{i}^{\prime}\right)$, if some prefix of $\left(z_{1}, \ldots, z_{i-1}\right)$ is in $B$ then $\left.Z_{i}^{\prime}\right|_{Z_{1}^{\prime}=z_{1}, \ldots, Z_{i-1}^{\prime}=z_{i-1}}$ is the uniform distribution $U_{m_{i}, q}$, otherwise $\left.Z_{i}^{\prime}\right|_{Z_{1}^{\prime}=z_{1}, \ldots, Z_{i-1}^{\prime}=z_{i-1}}=$ $\left.Z_{i}\right|_{Z_{1}=z_{1}, \ldots, Z_{i-1}=z_{i-1}}$. In the second case, $\left.X\right|_{Z_{1}=z_{1}, \ldots, Z_{i-1}=z_{i-1}}$ has min-entropy $k-\left(m_{1}+\cdots+\right.$ $\left.m_{i-1}\right)-\log _{q}(1 / \epsilon) \geq m_{i}$ since $\left(z_{1}, \ldots, z_{i-1}\right) \notin B$. In this case, $\left.Z_{i}^{\prime}\right|_{Z_{1}^{\prime}=z_{1}, \ldots, Z_{i-1}^{\prime}=z_{i-1}}$ is $\epsilon$-close to a distribution of min-entropy $k_{i}$ by Theorem 3.1 and the fact

$$
\left.Z_{i}^{\prime}\right|_{Z_{1}^{\prime}=z_{1}, \ldots, Z_{i-1}^{\prime}=z_{i-1}}=\left.Z_{i}\right|_{Z_{1}=z_{1}, \ldots, Z_{i-1}=z_{i-1}}=\operatorname{RSCon}_{n, m_{i}, q}\left(\left.X\right|_{Z_{1}=z_{1}, \ldots, Z_{i-1}=z_{i-1}}, Y_{i}\right) .
$$

In either cases $\left.Z_{i}^{\prime}\right|_{Z_{1}^{\prime}=z_{1}, \ldots, Z_{i-1}^{\prime}=z_{i-1}}$ is $\epsilon$-close to a distribution of min-entropy $k_{i}$. By Lemma C.3, $Z^{\prime}$ is $2 s \epsilon$-close to a $\left(k_{1}, \ldots, k_{s}\right) q$-ary block source.

It remains to prove that $Z$ is $s \epsilon$-close to $Z^{\prime}$, which implies that it is $3 s \epsilon$-close to a $\left(k_{1}, \ldots, k_{s}\right)$ $q$-ary block source. By Lemma C.1, for any $i \in[s]$, we have $\operatorname{Pr}\left[\left(Z_{1}, \ldots, Z_{i-1}\right) \in B\right] \leq \epsilon$. So the probability that $\left(Z_{1}, \ldots, Z_{i-1}\right) \in B$ for some $i \in[s]$ is bounded by $s \epsilon$. Note that the distribution $Z^{\prime}$ is obtained from $Z$ by redistributing the weights of $\left(z_{1}, \ldots, z_{s}\right)$ satisfying $\left(z_{1}, \ldots, z_{i-1}\right) \in B$ for some $i$. We conclude that $\Delta\left(Z, Z^{\prime}\right) \leq s \epsilon$, as desired.

Theorem 3.3 (from page 9). For any $\epsilon, \delta>0$, integer $m \geq 1$, and sufficiently large prime power $q \geq(n / \epsilon)^{O(1)}$, OuterSamp ${ }_{m, \delta, q}$ is an $(\epsilon, \delta)$ sampler of degree $t$ where $d=O(\log m), n=$ $O\left(m+\log _{q}(1 / \delta)\right)$ and $t=O\left(m^{2}+m \log _{q}(1 / \delta)\right)$.

Proof. We first show that OuterSamp $m, \delta, q$ is a $(4 m, \epsilon, q)$ extractor. Consider any random source $X$ over $\mathbb{F}_{q}^{n}$ with $q$-ary min-entropy $4 m$. Let $s, d_{i}$ be as in Definition 3.7. Let $k_{i}=4 \cdot 0.99 \cdot d_{i}$ for $i \in[s]$. Let $\epsilon_{0}=\frac{\epsilon}{4 s}$.

We have $\left(\sum_{i=1}^{s} 4 d_{i}\right)+\log _{q}\left(1 / \epsilon_{0}\right) \leq 4 m$ for sufficiently large $q \geq(n / \epsilon)^{O(1)}$. So by Theorem 3.2, $\mathrm{BlkCnvt}_{n,\left(4 d_{1}, \ldots, 4 d_{s}\right), q}$ is a $\left(4 m,\left(k_{1}, \ldots, k_{s}\right), 3 s \epsilon_{0}, q\right)$ block source converter. Therefore the distribution $\mathrm{BlkCnvt}_{n,\left(4 d_{1}, \ldots, 4 d_{s}\right), q}\left(X, U_{s, q}\right)$ is $3 s \epsilon_{0}$-close to a $\left(k_{1}, \ldots, k_{s}\right) q$-ary block source $X^{\prime}$. Then OuterSamp $_{m, \delta, q}\left(X, U_{d, q}\right)$ is $3 s \epsilon_{0}$-close to $\operatorname{BlkExt}\left(\operatorname{Line}_{2, q^{d_{1}}}, \ldots\right.$, Line $\left._{2, q^{d_{s}}}\right)\left(X^{\prime}, U_{1, q}\right)$.

By Lemma 3.2, Line $2, q^{d_{i}}$ is a $\left(k_{i} / d_{i}, \epsilon_{0}, q^{d_{i}}\right)$ extractor for $i \in[s]$ since $3+3 \log _{q^{d_{i}}}\left(1 / \epsilon_{0}\right) \leq 4 \cdot 0.99=$ $k_{i} / d_{i}$. Equivalently it is a $\left(k_{i}, \epsilon_{0}, q\right)$ extractor. By Lemma 3.1, BlkExt $\left(\operatorname{Line}_{2, q^{d_{1}}}, \ldots\right.$, Line $\left._{2, q^{d_{s}}}\right)$ is a 
$\left(\left(k_{1}, \ldots, k_{s}\right), s \epsilon_{0}, q\right)$ block source extractor. Therefore BlkExt $\left(\right.$ Line $_{2, q^{d_{1}}}, \ldots$, Line $\left._{2, q^{d_{s}}}\right)\left(X^{\prime}, U_{1, q}\right)$ is $s \epsilon_{0^{-}}$ close to $U_{m, q}$, which by the previous paragraph, implies that OuterSamp ${ }_{m, \delta, q}\left(X, U_{d, q}\right)$ is $4 s \epsilon_{0}$-close to $U_{m, q}$. By definition, OuterSamp $m, \delta, q$ is a $(4 m, \epsilon, q)$ extractor. By Theorem 2.1, it is also an $(\epsilon, \delta)$ sampler.

We have $d=s+1=O(\log m)$ and $n=O\left(m+\log _{q}(1 / \delta)\right)$. By Lemma 2.1, each Line ${ }_{2, q^{d_{i}}}$ has degree 2 as a manifold over $\mathbb{F}_{q}$. Therefore by Lemma 3.1, BlkExt $\left(\right.$ Line $_{2, q^{d_{1}}}, \ldots$, Line $\left._{2, q^{d_{s}}}\right)$ has degree $2^{s}$. By Theorem 3.2, BlkCnvt ${ }_{n,\left(4 d_{1}, \ldots, 4 d_{s}\right), q}$ has degree $n$. Therefore OuterSamp $m, \delta, q$ has degree $n 2^{s}=O\left(m^{2}+m \log _{q}(1 / \delta)\right)$.

Lemma 4.2 (from page 11). Let $S_{1}: \mathbb{F}_{q}^{n_{1}} \times \mathbb{F}_{q}^{d_{1}} \rightarrow \mathbb{F}_{q}^{d_{0}}$ be an $\left(\epsilon_{1}, \delta_{1}\right)$ sampler of degree $t_{1}$. And let $S_{2}: \mathbb{F}_{q}^{n_{2}} \times \mathbb{F}_{q}^{d_{2}} \rightarrow \mathbb{F}_{q}^{d_{1}}$ be an $\left(\epsilon_{2}, \delta_{2}\right)$ sampler of degree $t_{2}$. Then $S_{1} \circ S_{2}:\left(\mathbb{F}_{q}^{n_{1}} \times \mathbb{F}_{q}^{n_{2}}\right) \times \mathbb{F}_{q}^{d_{2}} \rightarrow \mathbb{F}_{q}^{d_{0}}$ is an $\left(\epsilon_{1}+\epsilon_{2}, \delta_{1}+\delta_{2}\right)$ sampler of degree $t_{1} t_{2}$.

Proof. Consider an arbitrary subset $A \subseteq \mathbb{F}_{q}^{d_{0}}$. Define $B(x)=\left\{z \in \mathbb{F}_{q}^{d_{2}}: S_{1}(x, z) \in A\right\}$ for each $x \in \mathbb{F}_{q}^{n_{1}}$. Pick $x_{1} \leftarrow U_{n_{1}, q}$ and $x_{2} \leftarrow U_{n_{2}, q}$. If $\left|\mu_{S_{1} \circ S_{2}\left(\left(x_{1}, x_{2}\right)\right)}(A)-\mu(A)\right|>\epsilon_{1}+\epsilon_{2}$ occurs, then either $\left|\mu_{S_{1}\left(x_{1}\right)}(A)-\mu(A)\right|>\epsilon_{1}$, or $\left|\mu_{S_{1} \circ S_{2}\left(\left(x_{1}, x_{2}\right)\right)}(A)-\mu_{S_{1}\left(x_{1}\right)}(A)\right|>\epsilon_{2}$ occurs. Call the two events $E_{1}$ and $E_{2}$ respectively.

Note that $E_{1}$ occurs with probability at most $\delta_{1}$ by the sampling property of $S_{1}$. Also note that

$$
\mu_{S_{1} \circ S_{2}\left(\left(x_{1}, x_{2}\right)\right)}(A)=\operatorname{Pr}_{y}\left[S_{1}\left(x_{1}, S_{2}\left(x_{2}, y\right)\right) \in A\right]=\operatorname{Pr}_{y}\left[S_{2}\left(x_{2}, y\right) \in B\left(x_{1}\right)\right]=\mu_{S_{2}\left(x_{2}\right)}\left(B\left(x_{1}\right)\right)
$$

whereas

$$
\mu_{S_{1}\left(x_{1}\right)}(A)=\operatorname{Pr}_{y}\left[S_{1}\left(x_{1}, y\right) \in A\right]=\underset{y}{\operatorname{Pr}}\left[y \in B\left(x_{1}\right)\right]=\mu\left(B\left(x_{1}\right)\right) .
$$

So the probability that $E_{2}$ occurs is $\operatorname{Pr}_{x_{1}, x_{2}}\left[\left|\mu_{S_{2}\left(x_{2}\right)}\left(B\left(x_{1}\right)\right)-\mu\left(B\left(x_{1}\right)\right)\right|>\epsilon_{2}\right]$ which is bounded by $\delta_{2}$ by the sampling property of $S_{2}$. By the union bound, the event

$$
\left|\mu_{S_{1} \circ S_{2}\left(\left(x_{1}, x_{2}\right)\right)}(A)-\mu(A)\right|>\epsilon_{1}+\epsilon_{2}
$$

occurs with probability at most $\delta_{1}+\delta_{2}$, as desired.

Finally, we have $\left.S_{1} \circ S_{2}\left(\left(X_{1}, X_{2}\right), Y\right)\right)=S_{1}\left(X_{1}, S_{2}\left(X_{2}, Y\right)\right)$ which has degree $t_{1} t_{2}$ in its variables $X_{1}, X_{2}, Y$ since $S_{1}$ and $S_{2}$ have degree $t_{1}$ and $t_{2}$ respectively. 